Research in Astron. Astrophys. 2015 Vol. X No. XX, 000-000

http://www.raa-journal.org http://www.iop.org/journals/raa

$\boldsymbol{R}$ esearch in

A stronomy and

A strophysics

\title{
The evolution of stellar metallicity gradients of the Milky Way disk from LSS-GAC main sequence turn-off stars: a two-phase disk formation history?
}

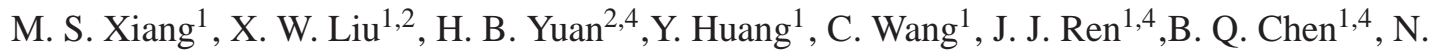 \\ C. Sun ${ }^{1}$, H. W. Zhang ${ }^{1}$, Z. Y. Huo ${ }^{3}$, A. Rebassa-Mansergas ${ }^{2,4}$ \\ ${ }^{1}$ Department of Astronomy, Peking University, Beijing 100871, P. R. China; xms@pku.edu.cn, \\ x.liu@pku.edu.cn \\ 2 Kavli Institute for Astronomy and Astrophysics, Peking University, Beijing 100871, P. R. China \\ 3 National Astronomical Observatories, Chinese Academy of Sciences, Beijing 100012, P. R. China \\ ${ }^{4}$ LAMOST Fellow
}

\begin{abstract}
Accurate measurements of stellar metallicity gradients in the radial and vertical direction of the disk and their temporal variations provide important constraints of the formation and evolution of the Milky Way disk. We use 297042 main sequence turn-off stars selected from the LAMOST Spectroscopic Survey of the Galactic Anti-center (LSSGAC) to determine the radial and vertical gradients of stellar metallicity, $\Delta[\mathrm{Fe} / \mathrm{H}] / \Delta R$, $\Delta[\mathrm{Fe} / \mathrm{H}] / \Delta|Z|$, of the Milky Way disk in the anti-center direction. We determine ages of those turn-off stars by isochrone fitting and measure the temporal variations of metallicity gradients. We have carried out a detailed analysis of the selection effects resulting from the selection, observation and data reduction of LSS-GAC targets and the potential biases of a magnitude limited sample on the determinations of metallicity gradients. Our results show that the gradients, both in the radial and vertical directions, exhibit significant spatial and temporal variations. The radial gradients yielded by stars of oldest ages ( $\gtrsim 11 \mathrm{Gyr})$ are essentially zero at all heights from the disk midplane, while those given by younger stars are always negative. The vertical gradients deduced from stars of oldest ages $(\gtrsim 11 \mathrm{Gyr})$ are negative and show only very weak variations with the Galactocentric distance in the disk plane, $R$, while those yielded by younger stars show strong variations with $R$. After being essentially flat at the earliest epochs of disk formation, the radial gradients steepen as age decreases, reaching a maxima (steepest) at age $7-8 \mathrm{Gyr}$, and then they flatten again. Similar temporal trends are also found for the vertical gradients. We infer that the assemblage of the Milky Way disk may have experienced at least two distinct phases. The earlier phase is probably related to a slow, pressure-supported collapse of gas, when the gas settle down to the disk mainly in the vertical direction. In the later phase, there are significant radial flows of gas in the disk, and the rate of gas inflow near the solar neighborhood reaches a maximum around a lookback time of $7-8 \mathrm{Gyr}$. The transition of the two phases occurs around a lookback time between 8 and $11 \mathrm{Gyr}$. The two phases may be responsible for the formation of the Milky Way thick and thin disks, respectively. And, as a consequence, we recommend that stellar age is a natural, physical criterion to distinguish thin and thick disk stars. From an epoch earlier than $11 \mathrm{Gyr}$ to one between 8 and $11 \mathrm{Gyr}$, there is an abrupt, significant change in magnitude of both the radial and vertical metallicity gradients, suggesting that stellar radial migration is unlikely to play an important role in the formation of thick disk.
\end{abstract}


Key words: Galaxy: abundances - Galaxy: disk - Galaxy: evolution - Galaxy: formation - techniques: spectroscopic

\section{INTRODUCTION}

The structure and evolution of the Galactic disk are key issues of the formation of the Milky Way, which serves as a unique window for the understanding of disk galaxy formation in general. With the unique advantage that the individual stars in the Milky Way can be resolved, it is possible to address the above issues via a detailed and comprehensive archeological approach.

It has been shown that the stellar density profile in the vertical direction of the Galactic disk is best fitted by the sum of two exponentials of different scale heights (e.g. Gilmore \& Reid 1983), suggesting that the Milky Way disk may contain two components: a thin and a thick disk. Similar two-component disks are also observed in external galaxies (Burstein 1979; Dalcanton \& Bernstein 2002; Yoachim \& Dalcanton 2008a,b; Elmegreen \& Elmegreen 2006). Further studies show that the thin and thick disks of the Milky Way are different not only in density profiles(Jurić et al. 2008; Cheng et al. 2012a), but also in kinematics, metallicities and ages. Compared to the thin disk stars, the populations of the thick disk are on average older (Fuhrmann 1998; Bensby et al. 2003, 2005; Reddy et al. 2006; Haywood et al. 2013), kinematically hotter (e.g. Soubiran et al. 2003), and more metal-poor but alpha-enhanced (e.g. Reddy et al. 2003, 2006; Bensby et al. 2003, 2005; Fuhrmann 2008). On the other hand, evidence has been presented suggesting that the thin and thick disks may not be two distinct components after all. For example, it is shown that the evolution of stellar populations resulted from a monotonically declining star formation rate can naturally reproduce the observed bimodality of the distribution of $\alpha$-element to iron abundance ratios $[\alpha / \mathrm{Fe}]$ (Schönrich \& Binney 2009a,b). Similarly, it has been suggested that the observed vertical density distributions of the individual populations selected in the $[\mathrm{Fe} / \mathrm{H}]-[\alpha / \mathrm{Fe}]$ plane ('mono-abundance populations' - MAPs) can be the result of internal evolution of the disk (Bovy et al. 2012). The question whether the Milky Way has two distinctive disks, and if so what their relation in term of origin remains inconclusive.

Various scenarios/models have been proposed in recent years to explain the formation of the Milky Way disk, especially the thick disks. Based on the observed stellar age - metallicity $-[\alpha / \mathrm{Fe}]$ relation, Haywood et al. (2013) proposed a two-phase disk formation scenario, in which the thick disk forms from well-mixed interstellar medium $8 \mathrm{Gyr}$ ago over a time scale of $4-5 \mathrm{Gyr}$, and it sets the initial chemical conditions for the later, slowly formed inner thin disk. Other models proposed to explain the presence of a thick disk invoke either external, violent processes or internal, secular evolution. In these models the thick disk results from, for example, 1) direct accretion of stars in infalling satellites (Abadi et al. 2003); 2) vertical heating of a pre-existing thin disk via minor mergers (Quinn et al. 1993; Villalobos \& Helmi 2008; Read et al. 2008; Kazantzidis et al. 2008, 2009; Purcell et al. 2009; Bird et al. 2012); 3) star formation during a rapid, turbulent disk phase of high gas accretion rate at early epoch (Brook et al. 2004, 2005; Bournaud et al. 2009); and 4) radial migration of stars (Roškar et al. 2008; Schönrich \& Binney 2009b; Loebman et al. 2011). Those models reproduce well some aspects of the observations, yet it remains unclear which model(s) is the most realistic one. It is no doubt that the predictions of some of the aforementioned models sensitively depend on the input assumptions that are often poorly constrained-for instance, the gas infall/inflow rate and the frequency of stellar migration in the radial migration model of Schönrich \& Binney (2009b). Comprehensive and accurate observations are therefore crucial to better constrain those models.

Over the lifetime of a low mass star, the surface metallicity of the star remains largely the same as that at the time of formation. Stellar metallicity thus serves as a fossil record of the chemical conditions of the Galaxy at the time the star was born. By combining the metallicity and age information of a large sample of stars, it is possible to reveal the Galaxy formation and evolution history. The distribution of the stellar age and metallicity, as well as their spatial and temporal variations, contain important information of the past history of gas infall, accretion and inflow, as well as of the possible merger events and the 
stellar dynamic evolution (e.g. Larson 1976; Quinn et al. 1993; Schonrich \& Binney; 2009a,b). This thus serves as a powerful tool to constrain the various scenarios/models of Galactic disk formation.

On the one hand, different models/scenarios often predict different distributions of stellar age and metallicity, as well as kinematics. For example, a thick disk formed via a rapid, gas-rich merger at early epoch has a narrow age distribution and almost no vertical metallicity gradients (Brook et al. 2004, 2005), while a thick disk formed from a slow, pressure-supported collapse, following the formation of the extreme Population II stars, has an intermediate age distribution and shows negative vertical metallicity gradients (Larson 1976; Gilmore et al. 1989). A thick disk formed by heating a pre-existing thin disk as a result of minor mergers should contain stars older than the age of the current thin-disk (e.g. Quinn et al. 1993), while a thick disk arising from stellar radial migration should contain stars of a wide range of age distribution (e.g. Schonrich \& Binney 2009b). On the other hand, many of the aforementioned models are proposed to explain some specific aspects of the disk formation, rather than to provide comprehensive predictions of the disk properties, including age, metallicity and kinematics. It is thus not easy to implement a comprehensive test of those models with observations, especially considering the model predictions often sensitively depend on the input assumptions. A good knowledge of the stellar age and metallicity distributions of a large sample of stars is helpful to set the baseline of the models, so as to further tighten their predictions to be tested with observations.

Metallicity gradients of the Galactic disk have been measured from various tracers, including those of relatively young ages, such as OB stars (e.g. Daflon \& Cunha 2004), Cepheid variables (e.g. Andrievsky et al. 2002; Luck et al. 2006; Yong et al. 2006), H II regions (e.g. Balser et al. 2011) and open clusters (e.g. Friel 1995; Friel et al. 2002; Chen et al. 2003; Magrini et al. 2009), and those of intermediate-to-old ages, such as planetary nebulae (PNe; Maciel \& Quireza 1999; Costa et al. 2004; Henry et al. 2010), FGK dwarfs (e.g. Katz et al. 2011; Cheng et al. 2012b; Boeche et al. 2013), horizontal branch stars (Chen et al. 2010, 2011; Bilir et al. 2012) and red giants (Hayden et al. 2014). In general, the studies yield negative gradients in both the radial and vertical directions of the disk, along with some fine structures. Significant scatters amongst the results, however, do exist. Young populations often yield a linear radial gradient of values between -0.01 and -0.09 dex kpc${ }^{-1}$ (e.g. Lemasle et al. 2013), and there are also evidence that a linear function is not sufficient to describe the observed radial variations of metallicity in the whole disk (e.g. Luck et al. 2003, 2011; Andrievsky et al. 2004; Lépine et al. 2011). From an analysis of the relatively old F turn-off stars targeted in the SEGUE survey (Yanny et al. 2009), Cheng et al. (2011b) show that the radial metallicity gradient flattens with increasing $|Z|$, the absolute height above the disk plane, and becomes completely flat at $|Z|>1 \mathrm{kpc}$, a result that seems to support the rapid, highly turbulent gas-rich merger origin of the thick disk at high redshifts (Brook et al. 2004, 2005). Similar trend of variations of radial metallicity gradient is also found by Boeche et al. (2013) from dwarf stars observed by the RAVE survey (Steinmetz et al. 2006) and in the GenevaCopenhagen survey (GCS; Nordstörm et al. 2004), as well as by Hayden et al. (2014), from red giants targeted in the APOGEE survey (Majewski et al. 2010). In the vertical direction of the disk, negative gradients are found with the exact value varies from study to study (e.g. $-0.068 \mathrm{dex} \mathrm{kpc}^{-1}$, Katz et al. $2011 ;-0.22 \mathrm{dex} \mathrm{kpc}^{-1}$, Chen et al. $2011 ;-0.14 \mathrm{dex} \mathrm{kpc}^{-1}$, Kordopatis et al. $2011 ;-0.30 \mathrm{dex} \mathrm{kpc}^{-1}$, Schlesinger et al. 2012; $-0.243 \mathrm{dex} \mathrm{kpc}^{-1}$, Schlesinger et al. 2014). A strong dependence of the vertical gradient on the Galactocentric distance is also found (Hayden et al. 2014).

To interpret those results, it is helpful to keep several things in mind. Firstly, the intrinsic spatial distributions of different tracers probably differ significantly. For example, young stars are mostly restricted to low Galactic latitudes while older stars are much more spread out. Secondly, given that different tracers usually have different characteristic ages, they actually probe metallicity gradients of different evolutionary epochs of the Galaxy. Thirdly, different studies make use of data from observations of different probe limits (in both magnitude and volume) and target selection functions. Those effects are responsible, at least partially, for the different results obtained in various studies. A complete spectroscopic data sample, containing stars that spread over the whole age range of the Galaxy, and, at the same time, having a large enough probe volume as well as number density for any given age, would be extremely useful to avoid/reduce any potential selection effects and draw a clear picture of the chemical enrichment history of the Galactic disks. 
Obtaining a statistically complete spectroscopic sample of disk stars is, however, an extremely difficult task. One obvious obstacle is that the stars are widely distributed over the whole sky because of our own location in the Galactic disk, thus one needs a survey with extremely large sky coverage to target those stars. Secondly, restricted by limited observing capability, only a tiny fraction of the numerous stars have been spectroscopically targeted hitherto. Finally, disk stars often suffer from significant amount of extinction by the interstellar dust grains, and, as a consequence, reaching out spectroscopically to a substantial distance in the disk is an extremely challenging task. Available large sky area spectroscopic surveys are either restricted to the solar neighborhood or geared toward halo directions in order to avoid the disk.

The LAMOST Spectroscopic Survey of the Galactic Anti-center (LSS-GAC; Liu et al. 2014) aims to collect medium-to-low resolution $(R \sim 1800)$ optical spectra $(3700<\lambda<9000 \AA)$ of more than 3 million stars down to a limiting magnitude of $17.8 \mathrm{mag}$ (in $r$-band) in a approximately 3400 sq.deg. contiguous sky area in the Galactic anti-center direction, making use of the newly built LAMOST spectroscopic survey telescope (Cui et al. 2012). The survey will deliver accurate stellar parameters (radial velocity $V_{\mathrm{r}}$, effective temperature $T_{\text {eff }}$, surface gravity $\log g$, metallicity $[\mathrm{Fe} / \mathrm{H}]$, and $\alpha$-element to iron abundance ratio $[\alpha / \mathrm{Fe}]$ ) derivable from the collected spectra. The contiguous sky coverage, large number of stars and high sampling density (100 - 200 stars per squared degree), as well as the specifically designed simple yet nontrivial target selection algorithms [random selection in the $(g-r)-r$ and $(r-i)-r$ Hess diagrams; Liu et al. 2014; Yuan et al. 2015] make the LSS-GAC particularly suitable to study the Galactic disk in a star by star yet statistically meaningful manner. The LSS-GAC was initiated in September 2012, and is expected to complete in 2017. The first LAMOST official data release of the whole LAMOST survey (LAMOST DR1; Luo et al. 2015), as well as the first release of value-added catalog of LSS-GAC (Yuan et al. 2015), have been recently publicly released. The latter includes stellar parameters yielded by the LAMOST Stellar Parameter Pipeline at Peking University (LSP3; Xiang et al. 2015b), as well as values of interstellar extinction and stellar distance estimated with a variety of techniques, for a sample of almost another 700000 stars targeted by the LSS-GAC by 2013.

In this work, we define a sample of about 300000 main sequence turn-off (MSTO) stars selected from the first release of value-added catalog of LSS-GAC in an effort to study the age-dependent metallicity gradients of the Galactic disk. The paper is organized as follows. In Section 2, we briefly introduce the value-add catalog of LSS-GAC. In Section 3, we introduce the MSTO star sample in detail. In Section 4, we characterize the sample selection effects. We present our results in Section 5, followed by a discussion of the implications as well as potential uncertainties of our results in Section 6. A summary is presented in Section 7.

\section{THE VALUE-ADDED CATALOG OF LSS-GAC}

Most stars in the current sample are observed during the Pilot- (Oct. 2011 - Jun. 2012) and first-twoyear (Oct. 2012 - Jun. 2014) Regular Surveys of LSS-GAC. Stellar parameters derived from spectra collected during the Pilot- and first-year Regular Surveys have already published in the first release of value-added catalog of LSS-GAC (LSS-GAC DR1; Yuan et al. 2015). Data from the second-year Regular Survey will be publicly available in the second release of value-added catalog of LSS-GAC (LSS-GAC DR2; Xiang et al. in prep.).

The LSS-GAC DR1 contains stellar parameters derived from spectra of signal-to-noise ratios (SNRs) better than 10 for 664773 stars, including 219045 stars from the BMF (Bright, Median-bright, Faint) plates toward the Galactic Anti-center $(150<l<210$ deg. and $|b|<30$ deg. $), 52921$ stars in the M31/M33 area, and 392807 stars from the VB (Very Bright; $r<14$ mag) plates (Liu et al. 2014; Yuan et al. 2015). The stellar parameters are derived with the LSP3 (Xiang et al. 2015b), and have an overall accuracy of $5-10 \mathrm{~km} \mathrm{~s}^{-1}, 150 \mathrm{~K}, 0.25 \mathrm{dex}, 0.15 \mathrm{dex}$ in $V_{\mathrm{r}}, T_{\mathrm{eff}}, \log g$ and $[\mathrm{Fe} / \mathrm{H}]$, respectively, for a blue-arm spectral SNR better than 10 per pixel. The blue-arm spectral SNR is defined as the median value in the wavelength range $4600-4700 \AA$, and one pixel corresponds to $1.07 \AA$ at $4650 \AA$. The LSSGAC DR1 also includes values of extinction and distance of individual stars, estimated with a variety of methods. Typical uncertainty is 0.04 mag for the estimates of color-excess $E(B-V), 15,10$ and 30 per 


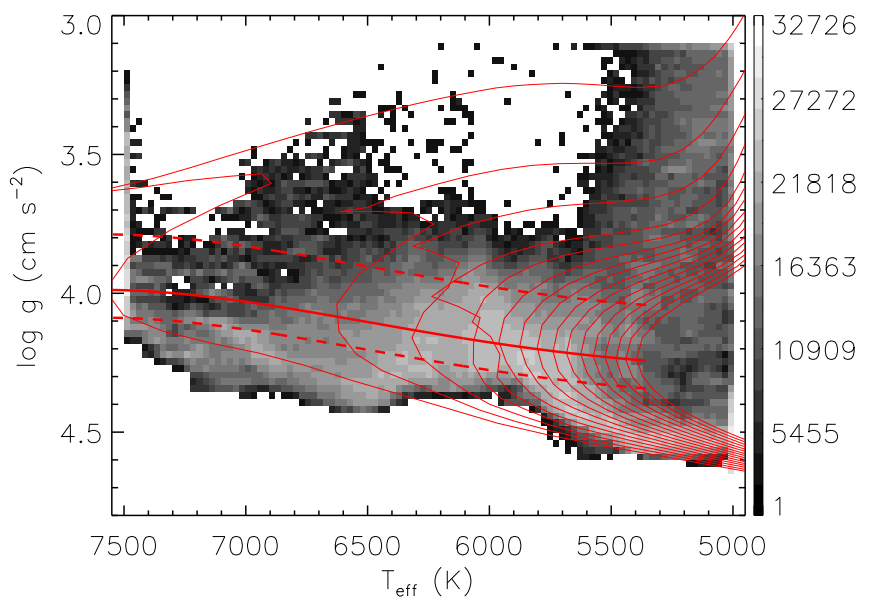

Fig. 1 Trajectories of MSTO stars of ages, from left to right, 1 to $16 \mathrm{Gyr}$, in the $T_{\text {eff }}-\log g$ plane deduced from the $\mathrm{Y}^{2}$ isochrones of $[\mathrm{Fe} / \mathrm{H}]=0$ and $[\alpha / \mathrm{Fe}]=0$. The dashed lines delineate the area of MSTO stars. The background grey-scale image shows the number density of stars of spectral SNR higher than 15 of the current sample.

cent respectively for distances of dwarfs, red-clump stars and giants (Yuan et al. 2015). The LSS-GAC value-added catalog for the second-year Regular Survey contains stellar parameters as well as extinction and distance of about another 700000 stars with a spectral SNR higher than 10.

In the current study, we have also included $~ 80000$ stars observed by the Galactic spheroidal parts of the LEGUE survey (Zhao et al. 2012; Deng et al. 2012). Those stars have high Galactic latitudes thus suffer little extinction. They were originally analyzed to examine the accuracy of the flux calibration pipeline of LSS-GAC (Xiang et al. 2015a). The stellar parameters of those stars are also measured with the LSP3, and their values of extinction and distance are estimated in the same way as for the LSSGAC targets. Finally, the current sample also contains 50000 stars in the Kepler field targeted by the LAMOST-Kepler Project (De Cat et al. 2015). Those stars are also processed in the same way as for LSS-GAC targets.

\section{THE MAIN SEQUENCE TURN-OFF STAR SAMPLE}

We define a MSTO star sample that contains stars of $\log g$ around the main sequence turn-off. The effective temperature of an MSTO star is sensitive to stellar age so that it can be used to obtain relatively reliable estimate of the latter using the technique of stellar isochrone fitting.

\subsection{Sample definition}

To select MSTO stars, we first define the ranges of values of $T_{\text {eff }}$ and $\log g$ of MSTO stars of different ages for a given set of $[\mathrm{Fe} / \mathrm{H}]$ and $[\alpha / \mathrm{Fe}]$ by interpolating stellar isochrones. As an example, Fig. 1 shows the trajectories of MSTO stars deduced from the Yonsei - Yale ( $\mathrm{Y}^{2}$ ) isochrones (Demarque et al. 2004) in the $T_{\text {eff }}-\log g$ plane for $[\mathrm{Fe} / \mathrm{H}]=0$ and $[\alpha / \mathrm{Fe}]=0$. For a given set of $T_{\text {eff }}$ and $[\mathrm{Fe} / \mathrm{H}]$, we first determine the value of $\log g$ of an exact MSTO star, $\log g_{\mathrm{TOF}}$. Then all stars with the given set of $T_{\text {eff }}$ and $[\mathrm{Fe} / \mathrm{H}]$ that have a value of $\log g$ within the range, $a<\log g-\log g_{\mathrm{TOF}}<b$, are selected as MSTO sample stars in the current analysis. The values of $a$ and $b$ are set to be -0.2 and 0.1 dex, respectively. A smaller value of $b$ than that of $a$ is adopted in order to reduce the potential contamination of dwarf stars. We further require that spectra of all stars in our sample have a SNR higher than 15, and have 


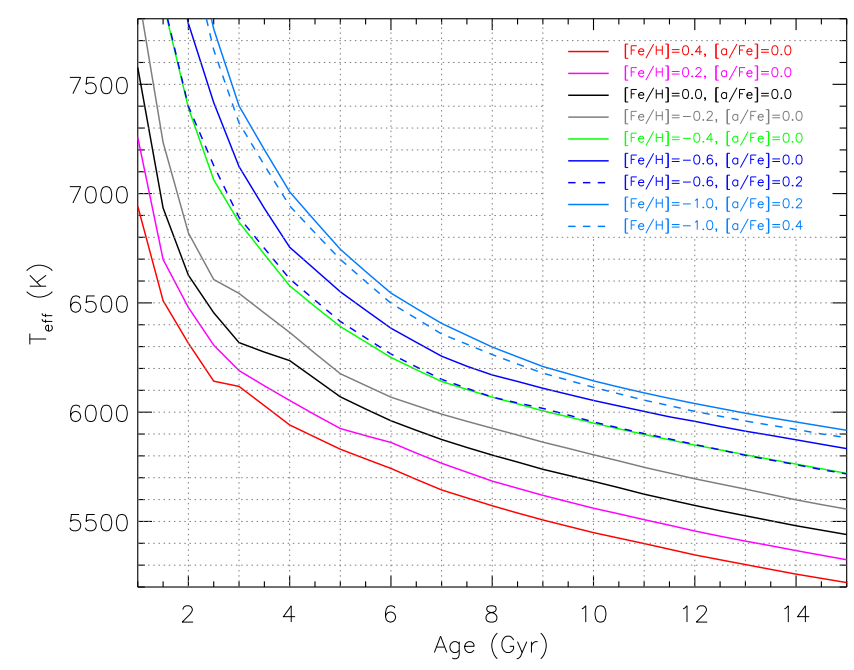

Fig. 2 Effective temperatures of MSTO stars deduced from the $\mathrm{Y}^{2}$ isochrones as a function of age for different sets of $[\mathrm{Fe} / \mathrm{H}]$ and $[\alpha / \mathrm{Fe}]$.

an effective temperature between 5400 and $7500 \mathrm{~K}$. The LSP3 yields more accurate stellar atmospheric parameters for stars of such SNRs and effective temperature range than for stars of lower spectral SNRs or of effective temperatures outside the range (Xiang et al. 2015b). In addition, as Fig. 2 shows, this effective temperature range encloses stars of age between 2 and $13 \mathrm{Gyr}$ for a wide range of metallicities, from $[\mathrm{Fe} / \mathrm{H}]=-1.0$ to +0.4 dex. A total of 338639 unique stars are selected with the above criteria.

\subsection{The stellar distance and age}

\subsubsection{Determinations of stellar distance and age by isochrone fitting}

We determine distances and ages of MSTO stars by isochrone fitting. Given the stellar atmospheric parameters $\left(T_{\text {eff }}, \log g,[\mathrm{Fe} / \mathrm{H}]\right.$ and $\left.[\alpha / \mathrm{Fe}]\right)$ of a star, the age and absolute magnitude of the star can be determined by interpolating stellar isochrones. Since values of $[\alpha / \mathrm{Fe}]$ are not available from the LSP3 yet, we have assumed that our sample stars follow the $[\mathrm{Fe} / \mathrm{H}]-[\alpha / \mathrm{Fe}]$ relation inferred for stars in the solar neighborhood (e.g. Venn et al. 2004). Stars of $[\mathrm{Fe} / \mathrm{H}]>0.0$ are assumed to have a constant $[\alpha / \mathrm{Fe}]$ of 0.0 dex; Stars of $-0.5<[\mathrm{Fe} / \mathrm{H}]<0.0$ are assumed to have an $[\alpha / \mathrm{Fe}]$ value that increases linearly with decreasing $[\mathrm{Fe} / \mathrm{H}]$ from 0.0 to 0.2 dex; Stars of $-1.0<[\mathrm{Fe} / \mathrm{H}]<-0.5$ are assumed to have an $[\alpha / \mathrm{Fe}]$ value that increases linearly with decreasing $[\mathrm{Fe} / \mathrm{H}]$ from 0.2 to $0.3 \mathrm{dex}$, while stars of $[\mathrm{Fe} / \mathrm{H}]<-1.0$ have a constant $[\alpha / \mathrm{Fe}]$ value of 0.3 dex. We have adopted the $\mathrm{Y}^{2}$ isochrones. As a test, the Dartmouth isochrones (Dotter et al. 2008) are also used for comparison. Stellar magnitudes of the $\mathrm{Y}^{2}$ isochrones are given in filter passbands of the Johnson-Cousin system (UVBRIJHK). We transfer them into the SDSS and 2MASS systems using the relations presented by Jester et al. (2005) and Carpenter (2001), respectively.

To deduce the distance, the magnitudes are corrected for extinction using $E(B-V)$ derived by comparing the measured photometric colors, $g-r, r-i, J-K_{\mathrm{s}}$, with synthetic values, as introduced in Yuan et al. (2015), and using the extinction coefficients of Yuan et al. (2013). For stars in the footprint of XSTPS-GAC, the optical band photometry are from the XSTPS-GAC survey (Liu et al. 2014), except for stars brighter than $13.0 \mathrm{mag}$, for which the data are taken from the UCAC4 catalog (Zacharias et al. 2013) based on the AAVSO Photometric All-Sky Survey (APASS; e.g. Munari et al. 2014), which 
is complete to $15 \mathrm{mag}$ in $r$-band (Henden \& Munari, 2014). For stars outside the footprint of XSTPSGAC, the optical magnitudes are taken from the UCAC4 catalog or the SDSS when available. In our sample, there are a few thousand stars that do not have optical photometry from either the XSTPS-GAC, the UCAC4 (APASS) or the SDSS surveys. For those stars, only the 2MASS $J, H, K_{\mathrm{s}}$ magnitudes are used when deriving values of extinction. The algorithm used to derive stellar distances is similar to that introduced in Yuan et al. (2015). The only difference is that, in Yuan et al., the magnitudes of a star on an isochrone that has stellar atmospheric parameters closest to those of the target star are simply adopted to estimate the distance of the latter, while in the current work, we have interpolated the isochrones to the desired parameters of our target star of concern. The differences in the resultant distances are however marginal in most cases.

\subsubsection{Calibration and uncertainties of the stellar distances}

There are several sources of distance errors, including errors of the adopted stellar atmospheric parameters, uncertainties of the stellar (atmospheric) models that yield the isochrones, and any potential mismatches in absolute scale between the LSP3 stellar atmospheric parameters and those of the isochrones.

We estimate the distance errors induced by random errors of the LSP3 stellar atmospheric parameters by comparing distances deduced from multi-epoch observations of duplicate stars in the LSS-GAC sample. There are 54881 pairs of duplicate observations of spectral SNRs better than 10 for which the SNRs of the pair spectra differ by less than 5. Those stars are divided into bins of SNR, $T_{\text {eff }}, \log g$ and $[\mathrm{Fe} / \mathrm{H}]$, and the mean and dispersion of distance differences yielded by the individual pairs of spectra in each bin are calculated. The mean values of differences are found to be very close to zero, while the dispersions vary with SNR and stellar atmospheric parameters, as one would expect. We thus construct a grid of distance errors, taken as the dispersion divided by square root of 2, as a function of SNR and stellar atmospheric parameters $\left(T_{\text {eff }}, \log g,[\mathrm{Fe} / \mathrm{H}]\right)$. For a star of given SNR and stellar atmospheric parameters, the distance error resulting from the random errors of LSP3 parameters is then estimated by interpolating the grid.

To quantify systematic errors induced by our method of distance estimation, we apply our method to stars in the MILES library (Sánchez-Blázquez et al. 2006) that have accurate Hipparcos distance measurements (Perryman et al. 1997). MILES is the template spectrum library used by LSP3 to deduce stellar atmospheric parameters (Xiang et al. 2015b). Xiang et al. apply the LSP3 to MILES template spectra in order to quantify the systematic errors of the LSP3 algorithms. Here, when calculating the isochrone distances of MILES template stars, we have adopted the stellar atmospheric parameters deduced by applying the LSP3 to MILES template spectra. By this approach, one can calibrate the resultant distances against the Hipparcos measurements to correct for errors introduced by uncertainties of the isochrones and by systematic errors of the LSP3 parameters, as well as errors resulting from any potential mismatches in absolute scale between the LSP3 and isochrone stellar atmospheric parameters. There are 793 MILES stars with distance measurements available from the catalog of Extended Hipparcos Compilation (Anderson \& Francis 2012), 602 of them have distance errors smaller than 10 per cent. We divide the 602 stars into bins of $T_{\text {eff }}, \log g$ and $[\mathrm{Fe} / \mathrm{H}]$. The bin size is adjustable to ensure that in each bin there are sufficient number of stars. Normally, we require that each bin contains at least 20 stars. An upper limit of $600 \mathrm{~K}, 0.4$ dex and 1.2 dex is set for bin size in $T_{\text {eff }}, \log g$ and $[\mathrm{Fe} / \mathrm{H}]$ , respectively. As a result, a small number of bins have less than 20 stars. The relatively large bin size in $[\mathrm{Fe} / \mathrm{H}]$ is designed to account for the relatively small number of very metal-poor stars. For bins containing more than 5 stars, the mean and dispersion of differences between the isochrone and Hipparcos distances are calculated. Note that for F/G-type stars having typical disk star metallicities (e.g. [Fe/H] $<-0.6)$, as of interest to this work, there are about 20 or more stars in each bin. We select bins of $4500<T_{\text {eff }}<7500 \mathrm{~K}, \log g>3.4$ dex and $[\mathrm{Fe} / \mathrm{H}]>-1.5$ dex that encompass the parameter space of all stars in our MSTO sample, and fit the means and dispersions of differences as a function of $T_{\text {eff }}, \log g$ and $[\mathrm{Fe} / \mathrm{H}]$ using a third-order polynomial. The mean differences as a function of stellar parameters are adopted as the systematic errors of our distance estimates, and is corrected for to yield our final distance 
estimates. The dispersions, after combining with the random errors estimated above using LAMOST duplicate observations, are adopted as the final errors of our distance estimates.

\subsubsection{Calibration and uncertainties of the stellar age}

Errors of age estimates are determined by Monte-Carlo simulations that propagate errors of stellar atmospheric parameters as a function of SNR, $T_{\text {eff }}, \log g$ and $[\mathrm{Fe} / \mathrm{H}]$, including both random and systematic errors.

As described in Xiang et al. (2015b), the systematic errors actually result from two main causes, one is the inadequacy of the LSP3 algorithms - for instance, the boundary effects when estimating $\log g$. The other is the uncertainties in stellar atmospheric parameters of the MILES templates. The first cause induces offsets to stellar atmospheric parameters derived with LSP3, and the magnitudes of the offsets vary with location in the parameter space, while the second cause induces dispersions to the LSP3 stellar atmospheric parameters. The systematic errors are estimated by comparing the MILES stellar atmospheric parameters and those derived with LSP3 from the MILES template spectra. Different to the approach of Xiang et al. (2015b), where the errors induced by the above two causes are treated as a whole to derive the final systematic errors, here we determine errors induced by the two causes separately. The offsets induced by the first cause are determined in a similar manner to that has been carried out for the distance errors as introduced above. The LSP3 adopts the weighted mean (for $T_{\text {eff }},[\mathrm{Fe} / \mathrm{H}]$ ) or biweight mean (for $\log g$ ) parameters of the $n$ templates best-matching the target spectrum. Here $n$ is larger than 10 for the $T_{\text {eff }}$ and $[\mathrm{Fe} / \mathrm{H}]$ estimation, and $n=8$ for $\log g$ estimation. Thus systematic errors induced by the second cause can be expressed as $\sqrt{\sigma^{2}\left(X-X_{0}\right) / N}$, where $X$ represents the stellar atmospheric parameters derived with LSP3 for the MILES templates, $X_{0}$ represents the MILES stellar atmospheric parameters, and $N$ is a number between 1 and $n$. Here, we assume that $N=4$. These dispersions are also determined in a similar manner to that has been carried out for the distance errors as introduced above. The dispersions, combining with the random errors deduced by comparing results from duplicate observations as described in Xiang et al. (2015b), are adopted to be the error estimates of the stellar atmospheric parameters in the current work. The errors are functions of spectral SNR and the stellar atmospheric parameters themselves.

In order to use Monte-Carlo simulations to estimate errors of our age determinations, we create a dense grid in the parameter space $\left(T_{\text {eff }}, \log g\right.$ and $\left.[\mathrm{Fe} / \mathrm{H}]\right)$. For each node, random errors are assigned to parameters $T_{\text {eff }}, \log g$ and $[\mathrm{Fe} / \mathrm{H}]$, assuming Gaussian error distributions, with the dispersions of distributions given by the above error estimates. With the error-added parameters, $T_{\mathrm{eff}}, \log g$ and $[\mathrm{Fe} / \mathrm{H}]$, the age is then determined by isochrone fitting. The experiment is repeated for 3000 times for each grid point. The deviation of the mean age yielded by the 3000 simulations from the desired age that corresponds to the grid parameters $T_{\text {eff }}, \log g$ and $[\mathrm{Fe} / \mathrm{H}]$, combining with offset of age caused by the offsets of LSP3 stellar atmospheric parameters as estimated above, is adopted as the systematic error of our age estimate, and is corrected for to yield the final age estimates. Note that the systematic errors of age arise from two sources, one is the systematic errors of stellar atmospheric parameters, and the other is the non-linear relation between the age and stellar atmospheric parameters and the uneven spacing of the theoretical isochrones. The age dispersion (standard deviation) yielded by the 3000 simulations is then adopted as the final errors of our age estimates. The errors are function of spectral SNR, $T_{\mathrm{eff}}, \log g$ and $[\mathrm{Fe} / \mathrm{H}]$.

A more robust estimation of systematic errors of our age estimates would require a direct comparison of our estimated ages with accurate measurements, such as those given by asteroseismological analysis. While such an effort is under way, here we concentrate on the estimation of relative, rather than absolute values of age.

To check the robustness (consistency) of our age estimates, we divide the MSTO sample stars into bins of $T_{\text {eff }}$ and $[\mathrm{Fe} / \mathrm{H}]$, and examine the median and dispersion of ages of stars in each bin. Similar exercise is also carried out for $T_{\text {eff }}$ and $[\mathrm{Fe} / \mathrm{H}]$, respectively. Fig. 3 plots the distribution of the resultant medians (a) and dispersions (d) of ages in the $T_{\text {eff }}-[\mathrm{Fe} / \mathrm{H}]$ plane, the medians (b) and dispersions (e) of $[\mathrm{Fe} / \mathrm{H}]$ in the $T_{\text {eff }}$ - age plane, as well as the medians (c) and dispersions (f) of $T_{\text {eff }}$ in the age 

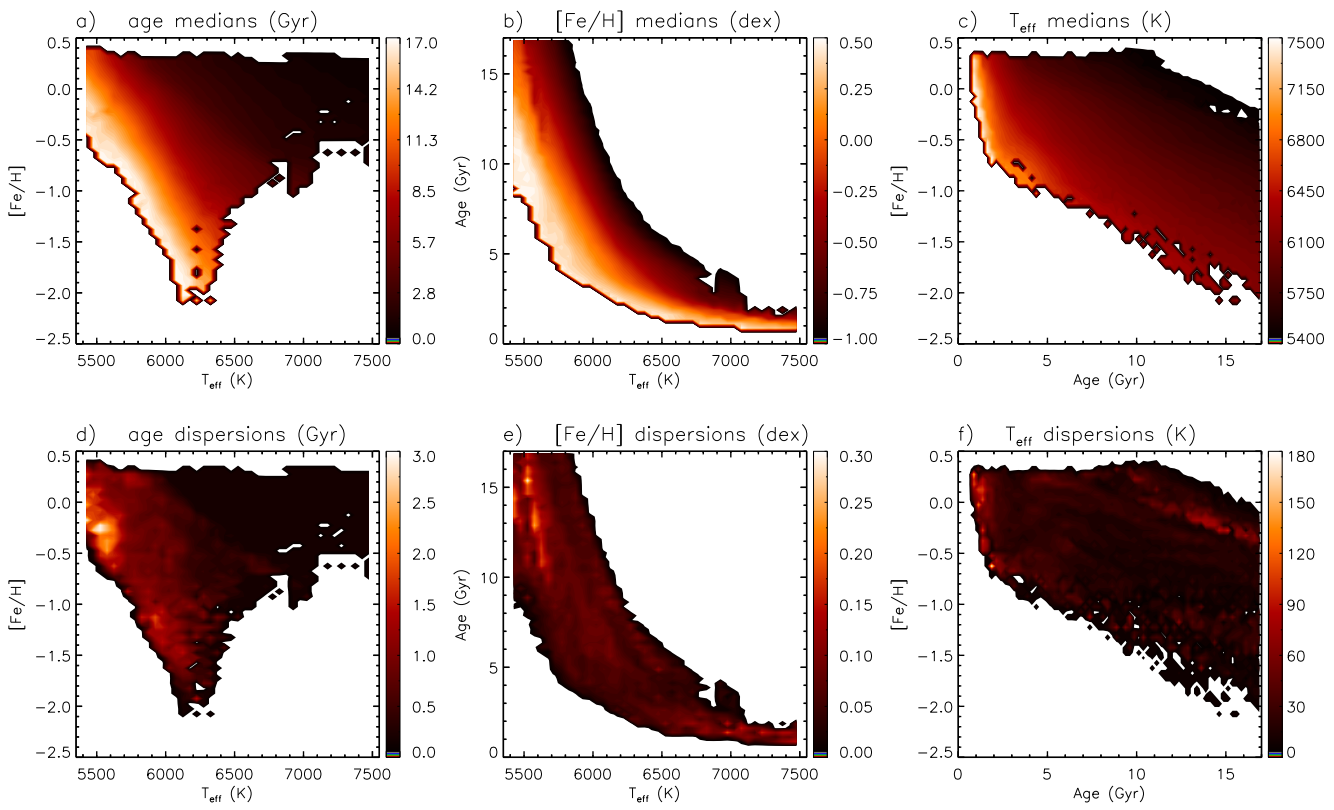

Fig. 3 Robustness examine for the age estimates. From upper-left to bottom-right, the panels show respectively the distributions of a) age medians in the $T_{\text {eff }}-[\mathrm{Fe} / \mathrm{H}]$ plane; b) $[\mathrm{Fe} / \mathrm{H}]$ medians in the $T_{\text {eff }}$ - age plane; c) $T_{\text {eff }}$ medians in the age $-[\mathrm{Fe} / \mathrm{H}]$ plan; d) age dispersions in the $T_{\text {eff }}-[\mathrm{Fe} / \mathrm{H}]$ plane; e) $[\mathrm{Fe} / \mathrm{H}]$ dispersions in the $T_{\text {eff }}-$ age plane; and f) $T_{\text {eff }}$ dispersions in the age $-[\mathrm{Fe} / \mathrm{H}]$ plane.

- $[\mathrm{Fe} / \mathrm{H}]$ plane. The Figure shows clear trends of variations of the median values of each parameter. The dispersions are small in general, indicating that our method of age determination is robust. If we increase the value of parameter $b$ in Section 3.1, say from 0.1 to 0.15 , so as to include more stars in our MSTO sample, we find that while the trends of variations of the median values remain clearly visible, the dispersions become significantly larger at some locations of the parameter space as a result of contamination of dwarf stars which have large uncertainties of age estimates.

\subsubsection{Comparison with results deduced using the Dartmouth isochrones}

As a further check of our distance and age estimates, we apply the method using the Dartmouth isochrones (Dotter et al. 2008) to our sample stars, and compare the results with those deduced using the $\mathrm{Y}^{2}$ isochrones. Distances and ages deduced using the two sets of isochrones are compared in Fig. 4. The Figure shows that distances yielded by the two sets of isochrones are consistent well with each other. There is negligible systematic differences, with a dispersion of only a few per cent. While ages yielded by the $\mathrm{Y}^{2}$ isochrones are $1-2 \mathrm{Gyr}$ systematically younger than those deduced using the Dartmouth isochrones, with typical scatters of about 1 Gyr. Results for the age comparison are in agreement with the findings of Haywood et al. (2013). The systematic differences are probably caused by the different stellar physics and/or calibrations adopted in the calculation of the two sets of isochrones (Dotter et al. 2008). 

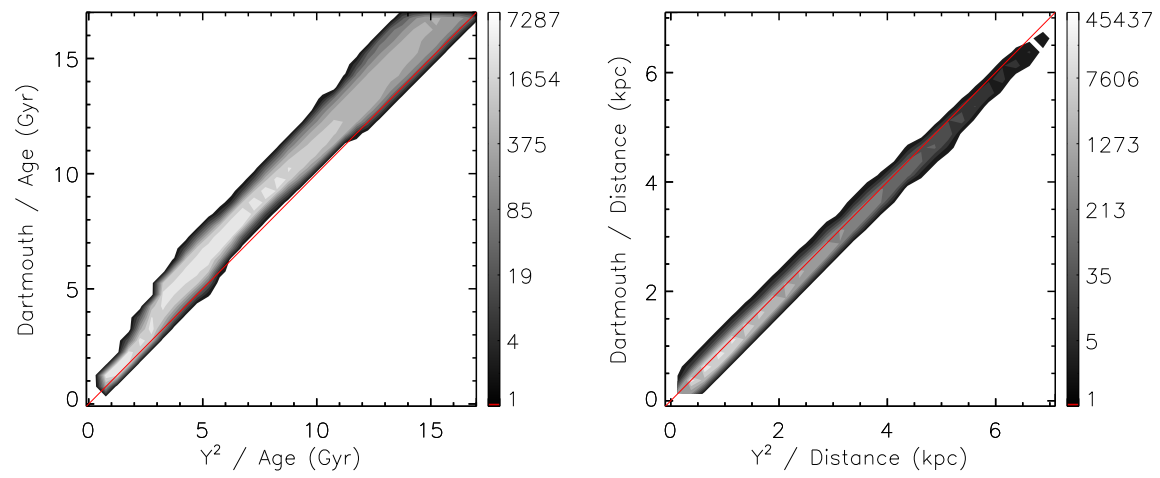

Fig. 4 Comparison of age (left) and distance (right) yielded by the $\mathrm{Y}^{2}$ isochrones with that by the Dartmouth isochrones for the MSTO sample stars. The grey scale contours show the stellar number densities on a logarithmic scale.
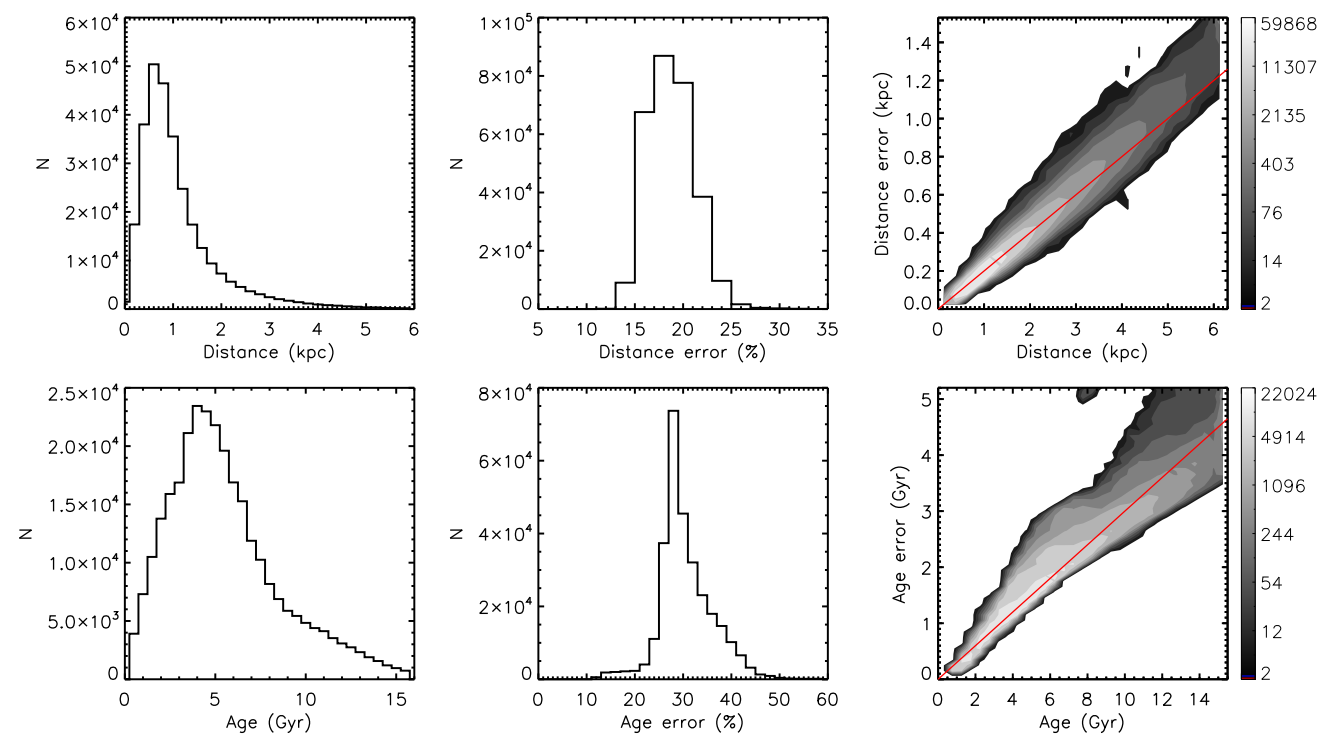

Fig. 5 Distribution of distances (upper-left), distance errors (upper-middle), ages (bottom-left) and age errors (bottom-middle) of the MSTO sample stars. The upper-right panel shows the stellar number density distribution in the distance and distance error plane, while the bottomright panel shows the distribution in the age and age error plane. The grey scale densities are plotted on a logarithmic scale.

\subsection{Distance, age and spatial position of the sample stars}

Fig. 5 plots the distributions of distances and ages, as well as their errors, of the MSTO sample stars. Most stars are within $2 \mathrm{kpc}$. Only about 35000 of them fall beyond. Typical distance errors are 20 per cent. The ages peak around $4-5 \mathrm{Gyr}$, with a tail extending beyond $8 \mathrm{Gyr}$. A small fraction of stars 

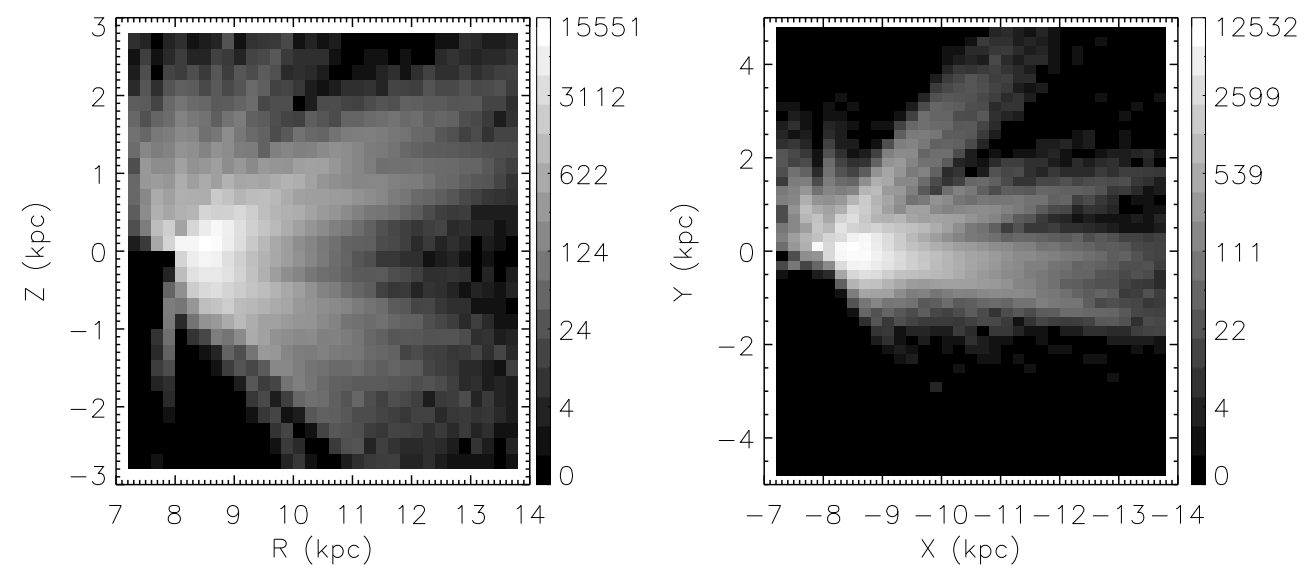

Fig. 6 Grey scale number density distribution of MSTO sample stars in the $R-Z$ and $X-Y$ planes. The stars are binned by $0.2 \times 0.2 \mathrm{kpc}$ in both the diagrams. The densities are shown on a logarithmic scale.

have ages older than 13 Gyr or even 15 Gyr. Although isochrones as old as 17 Gyr are available, stars that old are not expected given our knowledge that the universe is younger than 14 Gyr. Thus those old ages found for our sample are likely caused by the errors of stellar atmospheric parameters used. We have however opted to keep stars with determined ages as old as $16 \mathrm{Gyr}$ in our sample but discard those older than 16 Gyr. We believe that most of the rejected, only a small number $(\sim 2500)$, are actually cold dwarfs arising from some problematic $\log g$ estimates of LSP3. Typical age errors are 30 per cent.

Fig. 6 shows the number density distributions of sample stars in the $R-Z$ and $X-Y$ planes. Here $X, Y$ and $Z$ are coordinates of a right-handed Cartesian coordinate system with an origin at the Galactic center. $Z=0$ defines the Galactic disk plane. $X$ points toward the Galactic center, $Z$ toward the north Galactic pole. $R$ denotes Galactocentric distance in the disk $(X-Y)$ plane, i.e. $R=\sqrt{X^{2}+Y^{2}}$. The Figure shows that the MSTO sample stars cover well the regions between $7.5<R<13.5 \mathrm{kpc}$ and $-2.5<Z<2.5 \mathrm{kpc}$. Stars of high Galactic latitudes are found at larger values of $R$ due to less extinction they suffer from. In the disk plane, most stars fall between $-13.5<X<-7.5 \mathrm{kpc}$ and $-2<Y<4 \mathrm{kpc}$.

\section{SELECTION EFFECTS}

Since not all stars in a given volume are observed, one needs to consider the potential selection biases that may be presented in the sample and affect our results. The current sample has gone through two sets of main selection criteria. The first is imposed by the magnitude limit of the photometric surveys from which the LAMOST targets are selected and get observed. The magnitude limits are $9-14,14-$ 16.5, 16.5 - 17.8 mags in $r$-band for the LAMOST VB, B and M plates, respectively (Liu et al. 2014; Yuan et al. 2015). The second criteria are related with the SNR as well as $T_{\text {eff }}$ and $\log g$ cuts imposed when selecting the MSTO stars from the value-added catalog of LSS-GAC. We have thus implemented a two-steps approach to examine the selection effects. First, we calculate the fraction of stars selected from the photometric input catalogs that are targeted by the LAMOST and have a spectral SNR higher than 15. The calculation is carried out spectrograph by spectrograph in the color - magnitude diagram (CMD) $g-r$ verses $r$, as such a diagram is adopted to select the LSS-GAC targets for B, M and F plates. Note that the LSS-GAC target selection is in fact based on both $(g-r)-r$ and $(r-i)-r$ planes (Liu et al. 2014; Yuan et al. 2015), but here we adopt only the former to implement the calculation for simplicity. Such a simplification is not expected to significantly affect the results given that the stellar locus in the $(g-r)$ and $(r-i)$ plane is quite tight (e.g. Ivezić et al. 2007) and that we have divided the 


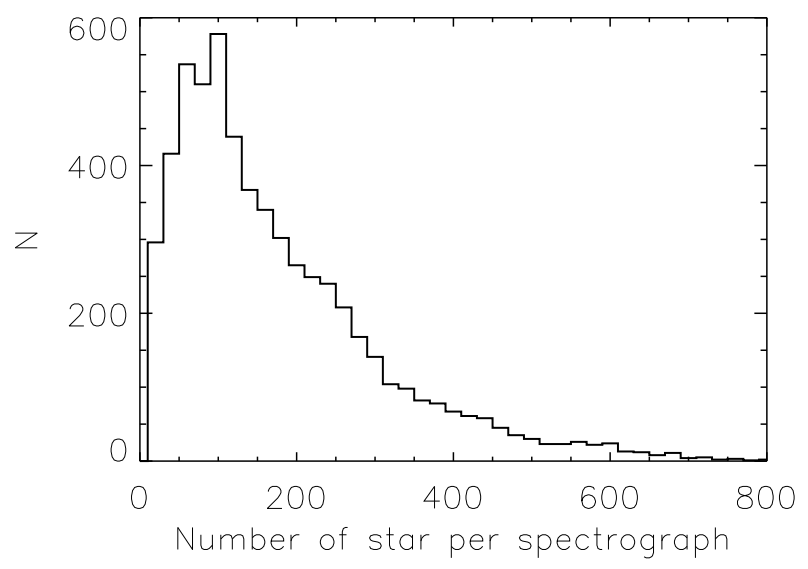

Fig. 7 Histogram of numbers of stars per spectrograph targeted that have a spectral SNR higher than 15, after combining plates of the same field (see the text).

stars into large color-magnitude cells to calculate the fraction (Section 4.1). Also note that although the selection of VB targets is not carried out in the $(g-r)-r$ plane but based on magnitudes of the targets only, our approach is still valid. The photometric input catalogs include the XSTPS-GAC, UCAC4 and SDSS catalogs. Secondly, we examine how the metallicity gradients deduced from the data are affected by the selection effects for a magnitude limited sample, utilizing mock data generated with Monte-Carlo simulations.

\subsection{CMD weights}

The total sample introduced in Section 2 contains 1130694 unique stars of all colors that have a spectral SNR higher than 15. The corresponding spectra are collected from 15967 spectrographs of 1126 plates of 418 fields (Yuan et al. 2015). For a given field, the projected positions on the sky of the 16 LAMOST spectrographs of different plates are nearly the same (differed by a few arc-seconds at most). We have thus combined together data from a specific spectrograph from different plates for the given field in order to increase the sampling densities. Spectrographs containing less than 10 stars are excluded, as well as those in which more than 20 per cent of the stars targeted have no counterparts in the aforementioned three photometric input catalogs, i.e. the XSTPS-GAC, UCAC4 and SDSS. The later cases occur in some few sky areas where the targets are selected from other catalogs (e.g. the 2MASS catalog or Kepler input catalog) and happen to have no complete photometric data from the XSTPS-GAC, UCAC4 and SDSS surveys. After excluding those spectrographs, 1052860 stars in 5911 spectrographs remain, 297042 of them are in our MSTO sample. Fig. 7 is a histogram of the numbers of stars per spectrograph which covers an FoV of $\sim 1$ sq.deg. The majority (3680) of spectrographs have more than 100 stars. The sampling rate (fraction) of a given field depend not only on the total number of stars observed in that field but also on the total number of stars in the field within the observed magnitude range. For fields of high Galactic latitudes in which only VB plates are observed, even a relatively small number of stars observed could imply a high completeness in the corresponding magnitude range. More than half spectrographs that have less than 100 stars are from fields of $|b|>30 \mathrm{deg}$.

For each spectrograph, we divide the stars into bins of $0.2 \times 0.5 \mathrm{mag}$ in the $(g-r)-r$ plane and for each cell calculate the fraction of stars targeted by the LSS-GAC with a resultant spectral SNR better than 15 with respect to the underlying photometric population. The inverse value of the fraction will 

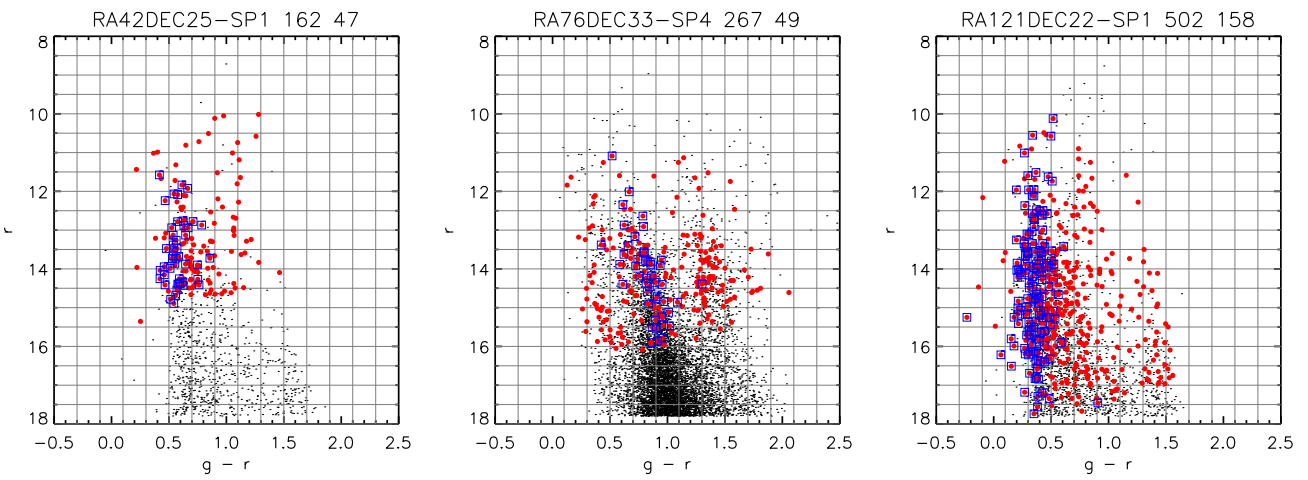

Fig. $8(g-r)$ verses $r$ CMD diagrams for three spectrographs from different fields. Black dots represent point sources in the photometric input catalogs of LSS-GAC, red dots are stars targeted by the LSS-GAC and have a spectral SNR higher than 15, and blue squares are MSTO stars. The field name and spectrograph ID, as well as the number of red dots and blue squares are marked on top of each panel.

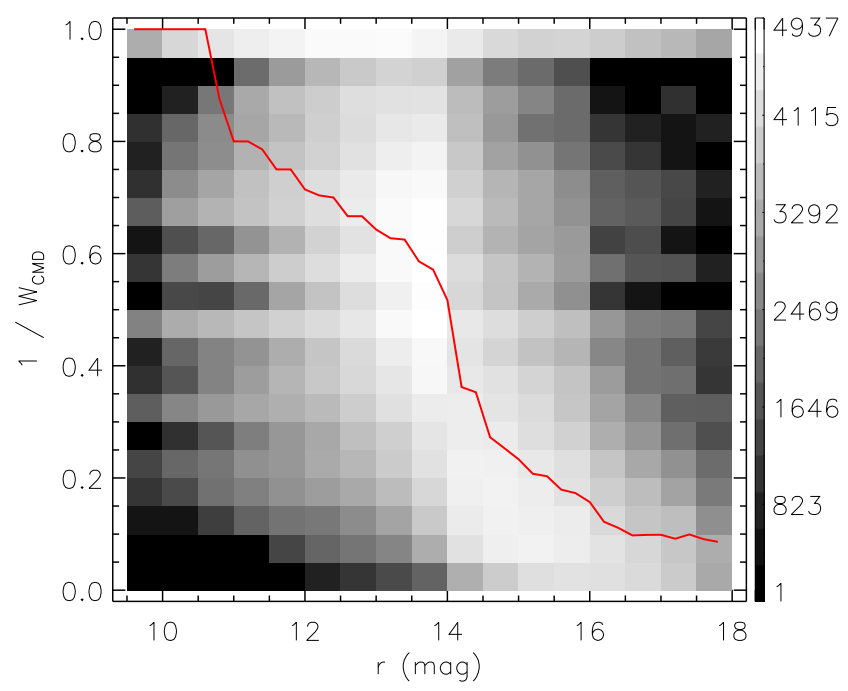

Fig. 9 Number density distribution in plane of $r$-band magnitude and the inverse of CMD weight for our MSTO sample stars. The grey scale is shown on a logarithmic scale. The red line shows the the median value of the inverse of CMD weights of the sample stars as a function of $r$-band magnitude.

be assigned as the CMD weights of sample stars in that cell when calculating the mean metallicities of the relevant spatial bins where the stars fall inside (see Section 5). This is illustrated in Fig. 8 for three spectrographs as an example. To obtain a reliable estimate of the fractions, it is essential that some sufficient number of stars are targeted in all CMD grid cells that may contain MSTO stars. This 
is not a problem when the number of stars observed in a given spectrograph is large enough, given the fact that the LSS-GAC is designed to target stars of all colors. However, it becomes a problem for spectrographs when the number of stars that meet the SNR requirement become to small. As a remedy, for spectrographs containing less than 50 stars with qualified spectra yet covering the whole magnitude ranges of both VB and $\mathrm{B}$ plates or $\mathrm{B}$ and $\mathrm{M}$ plates, the fractions are derived by combining data from all spectrographs of the same field instead of spectrograph by spectrograph. Finally, for some spectrographs, some CMD cells near the fainter end of the magnitude limit contain only one or two stars with qualified spectra, for example, the cell fainter than $16 \mathrm{mag}$ in the color bin of $0.7-0.9 \mathrm{mag}$ in the middle panel of Fig. 8. Such stars thus can not be used to represent the average metallicity of stars of the photometric sources in the corresponding CMD cells. To treat this problem, for those spectrographs, stars belonging to the faintest 2 per cent of all stars in the spectrograph of concern are set as outliers. Those stars will be assigned a minimum CMD weight of unity. Fig. 9 plots a grey scale contour map of stellar number density as a function of $r$-band magnitude and the inverse of thus derived CMD weight for all the sample stars. For the majority of stars, the inverse of CMD weights assigned decrease with increasing magnitude. This is due to the fact that in general, the fraction of sample stars relative to the underlying photometric population decreases with increasing magnitude. More than 90 per cent of the stars have an inverse of CMD weight larger than 0.1 which corresponds to a CMD weight smaller than 10 , suggesting that the sampling rate of our sample is high. The sampling rate is even much higher for the very bright $(r<14 \mathrm{mag}$ ) stars, for which half of them in the survey fields are included in our sample.

Applying the CMD weights to individual sample stars leads to a magnitude limited sample, although due to varying observing conditions and sensitivities of individual spectrographs, the limiting magnitudes differ from one sight line to another. Fig. 10 plots the limiting magnitudes at the bright and faint ends for the individual sight lines. The limiting magnitudes are derived spectrograph by spectrograph, after discarding the 2 per cent brightest as well as faintest sample stars in each spectrograph. The Figure shows that at the bright end, the majority of sight lines have a limiting magnitude brighter than $11.0 \mathrm{mag}$, while at the faint end, more than half sight lines have a limiting magnitude fainter than 16.5 mag. For sight lines for which no VB plates are observed, the bright end limiting magnitudes are fainter than 14.0 mag.

\subsection{Selection effects of a magnitude limited sample}

In this subsection, we examine potential selection effects that may affect the determinations of metallicity gradients using a magnitude limited sample. For this purpose, mock data are generated from MonteCarlo simulations of a model disk. The data are then used to investigate how the metallicity gradients derived from an observational sample deviate from the assumed true values as a result of the limiting magnitudes imposed on the sample.

Our simple model disk includes two stellar components, a thin and a thick disk. The mass density profile of each component is described by a double exponential,

$$
\rho(R, Z)=\rho\left(R_{\odot}, 0\right) \exp \left(-\frac{\left(R-R_{\odot}\right.}{R_{d}}\right) \exp \left(-\frac{|Z|+Z_{\odot}}{Z_{d}}\right),
$$

where $\rho$ is the mass density, $R_{\odot}$ and $Z_{\odot}$ are the position of the Sun in the radial and vertical direction of the disk, $R_{d}$ and $Z_{d}$ are the scale length and height of the disk, respectively. We adopt $\rho\left(R_{\odot}, 0\right)=$ $0.05 \mathrm{M}_{\odot} \mathrm{pc}^{-3}, R_{\odot}=8.0 \mathrm{kpc}$ (Reid 1993), $Z_{\odot}=25 \mathrm{pc}$ (Jurić et al. 2008). The scale parameters are from Juric et al. (2008), with $R_{d}=2.6 \mathrm{kpc}$ and $Z_{d}=0.3 \mathrm{kpc}$ for the thin disk, and $R_{d}=3.6 \mathrm{kpc}$ and $Z_{d}=$ $0.9 \mathrm{kpc}$ for the thick disk. For simplicity, we adopt a constant scale length and height for stars of different ages. The mass ratio of the thick disk to the thin disk at $R=8.0 \mathrm{kpc}$ and $Z=0 \mathrm{kpc}$ is adopted to be 0.12 (Jurić et al. 2008). We adopt the age-metallicity relation as adopted by a recent version of the Besancon Galactic model (Robin et al. 2003). In this model, the thick disk forms at $12 \mathrm{Gyr}$ ago, with a mean metallicity of $-0.48 \mathrm{dex}$ and a metallicity dispersion of $0.3 \mathrm{dex}$, while the thin disk forms continuously from $11 \mathrm{Gyr}$ ago to the current, with the mean metallicity increasing from -0.12 to $0.01 \mathrm{dex}$ at the current time and metallicity dispersion decreasing from 0.18 dex to $0.10 \mathrm{dex}$ at the present epoch. Note 

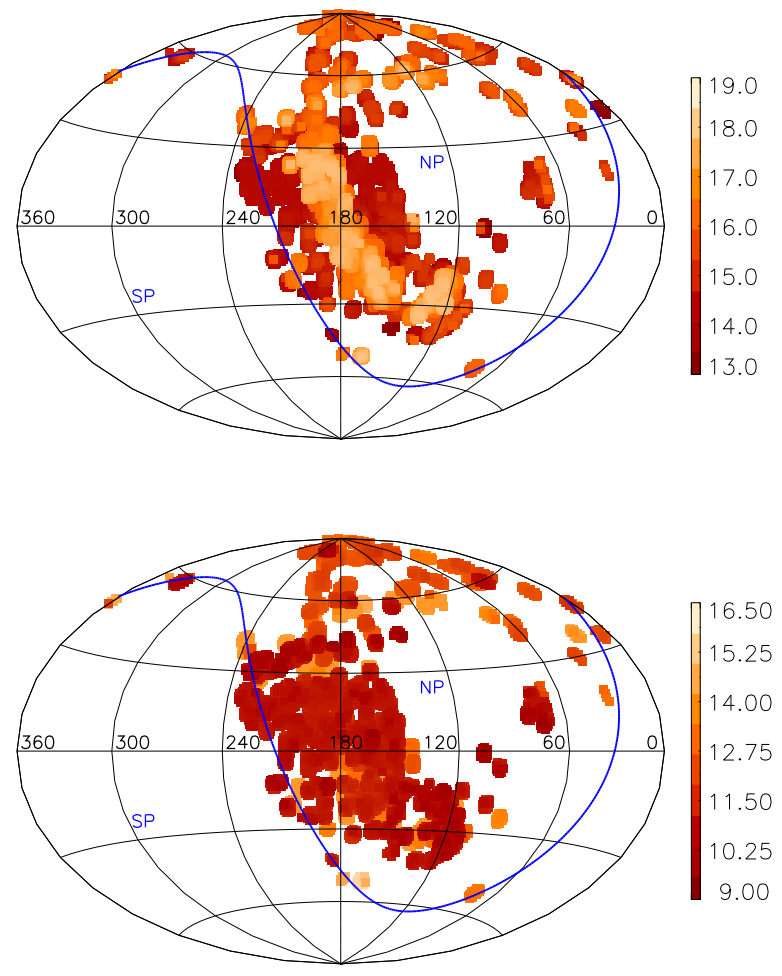

Fig. 10 Limiting magnitude at the faint (upper) and bright (lower) ends for the individual sight lines (spectrographs).

that this age-metallicity relation is taken from a version in the year of 2011 of the Besancon Galactic model, and it is different to the original one presented in Robin et al. (2003). In fact, we find that compared to the original one, the age-metallicity relation adopted here is actually better consistent with measurement based on our MSTO sample stars in the solar neighbourhood (Xiang et al. 2015, in prep.). Metallicity gradients in both the radial and vertical directions are assumed to be zero. We assume a constant star formation rate, and at a given age, the star formation follows the initial mass function (IMF) of Kroupa (2001). The $\mathrm{Y}^{2}$ isochrones are adopted to link the stellar physical properties with observables. We produce mock catalog with Monte-Carlo simulations, and for each star we add the interstellar extinction using the 3-dimensional extinction map of Chen et al. (2014). The map covers an area of about 6000 sq.deg. in the direction of Galactic anti-center. For stars outside of those area, the extinction map of Schlegel et al. (1998) is used. Finally, for each star, we assign a 25 per cent random error to its age, 20 per cent error to distance and 0.1 dex error to $[\mathrm{Fe} / \mathrm{H}]$.

We extract MSTO stars from the mock catalog that fall inside the FoVs and limiting magnitudes as our magnitude limited sample derived in the previous subsection in the anti-center direction $(R>8.0 \mathrm{kpc}$ ), and compare the metallicity gradients derived from the mock sample with the model assumptions (i.e. zero gradients). Fig. 11 plots the radial metallicity gradients thus derived from the mock sample as a function of absolute height above the Galactic plane $(|Z|)$ for stars of different ages. Details of the definition and derivation of radial metallicity gradients are introduced in Section 5.1. The 


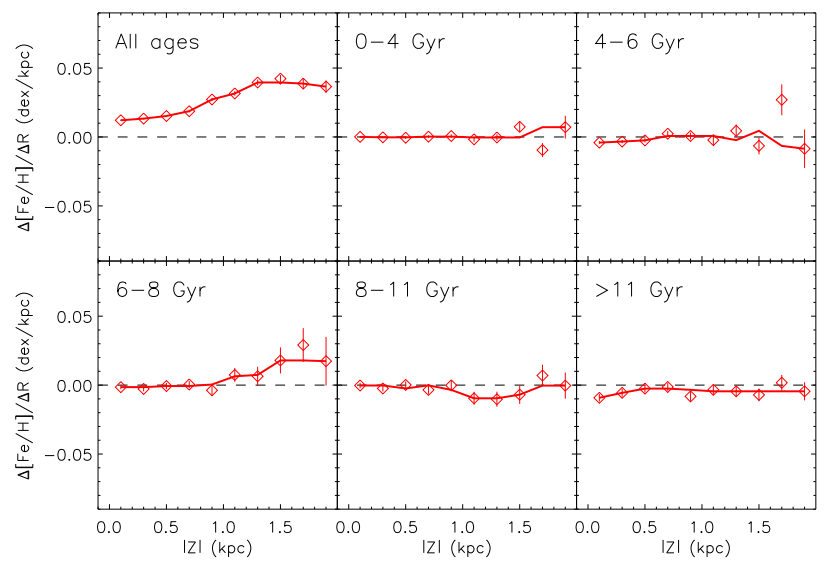

Fig. 11 Radial metallicity gradients as a function of $|Z|$ derived from the magnitude limited MSTO sample extracted from the mock catalog. The dashed line delineates the gradients (zero) of the model assumptions. The individual panels show results derived from stars of different age bins as marked in the plots.

Figure shows that gradients derived from stars of all ages deviate from the model input which is set at zero, marked by dashed lines in the plot. The deviations are artifacts caused by the different volumes probed by different stellar populations (ages and metallicities) for the given limiting magnitudes. As an example, this is illustrated in Fig. 12. Younger, more metal-rich stars have higher turn-off effective temperatures, thus higher (intrinsic) luminosities than older, more metal-poor stars. Given the limiting magnitudes imposed on the sample stars, younger, more metal-rich stars reach larger distances than those older, metal-poor ones, leading to an artificial positive metallicity gradient. The magnitude of this false gradient depends on the metallicity distribution of stars in the volume studied. As the height $(|Z|)$ increases, the fraction of metal-poor (thick disk) stars increases, yielding larger deviations. At $|Z|>1.5 \mathrm{kpc}$, metal-rich (thin disk) stars are so few such that they play unimportant role in the derivation of metallicity gradients. As a result, the deviation from the true value of the gradients derived actually decreases for heights above $2 \mathrm{kpc}$.

Fortunately, for sample stars in a narrow age bin, such artifacts are found to be almost absent. Fig. 11 shows that metallicity gradients derived from stars of the individual age bins reproduce well the underlying assumption for $|Z|<1.5 \mathrm{kpc}$. Here the age bins are defined in the same way as those for the observed MSTO star sample (Section 5.2). Note that in deriving the gradients, stars of $[\mathrm{Fe} / \mathrm{H}]$ $<-1.0$ dex have been discarded as for the case of the observed sample. The cut is to avoid contamination from halo stars (Section 5.1). At $|Z|<1.5 \mathrm{kpc}$, the largest (absolute) gradients derived from the mock data are about $0.01 \mathrm{dex} \mathrm{kpc}^{-1}$. The deviations from the true, zero gradient start to occur near the outer edges of height probed by the corresponding stellar populations. Near the edges, the selection biases induced by the metallicity effects, i.e, for a given age, more metal-rich stars are more luminous and are thus abundant in the observed sample, and vice versa, become important. At a given age, a 0.5 dex difference in metallicity may lead to about $0.2 \mathrm{mag}$ difference in absolute magnitude and about 10 per cent difference in distance, comparable to the bin size at $|Z| \sim 1.5-2 \mathrm{kpc}$. The selection effects are complicated given the fact that our sample have different limiting magnitudes for different sight lines. In our simulation, the maximum deviations of radial gradients are about $0.02-0.03 \mathrm{dex} \mathrm{kpc}^{-1}$, found for heights $1.5<|Z|<2 \mathrm{kpc}$ from stars in the 6-8 Gyr age bin (Fig. 11). Such deviations are unlikely to pose a major problem for our analysis given their small magnitudes and the fact that they occur only near the edges of volumes probed by the sample stars. Note that for our sample, bias induced by the bright 


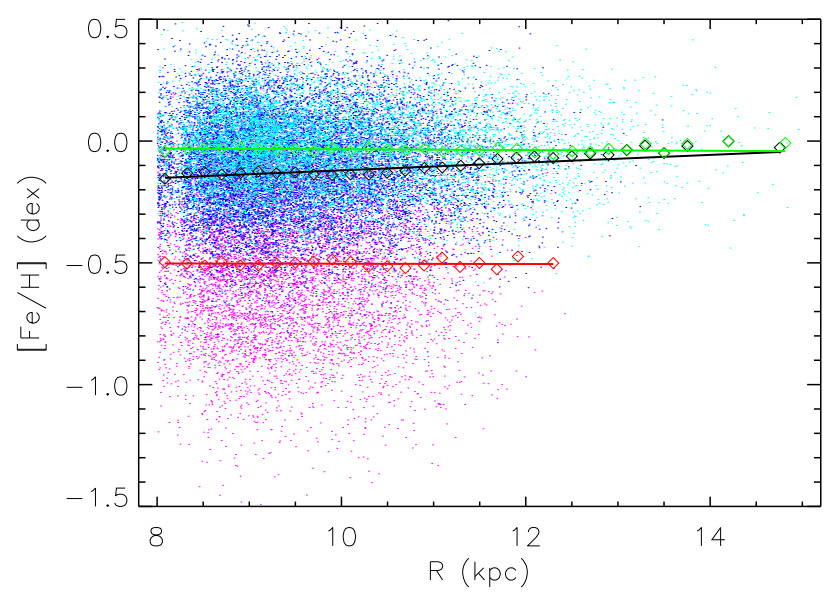

Fig. 12 The plot illustrates the selection effects that lead to false, non-zero radial metallicity gradients deduced from a magnitude limited sample of stars of $|Z|<0.1 \mathrm{kpc}$. The data are drawn from the mock catalog. Dots of different colors represent stars of different ages, with cyan ones being the youngest and red ones the oldest. Diamonds in green and red are respectively the mean metallicities of stars having an age of $2 \mathrm{Gyr}$ and $12 \mathrm{Gyr}$ at different Galactic radii, while black diamonds represent the mean metallicities of stars of all ages. The error bars associated with the mean metallicities are tiny and therefore not plotted. The lines are linear fits to the data points. Because of the limiting magnitudes imposed on the sample stars, there are few old, metal-poor stars at large distances.

end limiting magnitudes is negligible given that most FoVs of the sample contain very bright stars $(r \sim 10 \mathrm{mag})$. The maximum deviation caused by the bright end magnitude cut is about $0.01 \mathrm{dex} \mathrm{kpc}^{-1}$, as shown the first data point in the last panel (> 11 Gyr) of Fig. 11.

\section{RESULTS}

\subsection{Spatial distribution of mean metallicities}

We divide the sample MSTO stars into bins in the $R-Z$ plane. The bins step by 0.2 and $0.1 \mathrm{kpc}$ in the $R$ and $Z$ directions, respectively, with bin sizes determined by $0.2+\left|R-R_{\odot}\right| \times 0.1$ and $0.2+|Z| \times 0.1 \mathrm{kpc}$ in the $R$ and $Z$ directions, respectively, to account for distance errors which become larger at larger distances. For each bin, we calculate the mean metallicity, defined as,

$$
[\mathrm{Fe} / \mathrm{H}]_{\text {mean }} \equiv \int_{[\mathrm{Fe} / \mathrm{H}]_{1}}^{[\mathrm{Fe} / \mathrm{H}]_{2}} n([\mathrm{Fe} / \mathrm{H}]) \times[\mathrm{Fe} / \mathrm{H}] d[\mathrm{Fe} / \mathrm{H}]
$$

where $n([\mathrm{Fe} / \mathrm{H}])$ is the normalized, fractional stellar number density as a function of $[\mathrm{Fe} / \mathrm{H}],[\mathrm{Fe} / \mathrm{H}]_{1}$ is set to -1.0 dex to avoid contamination of halo stars, and $[\mathrm{Fe} / \mathrm{H}]_{2}$ is set to infinity. Note that the current sample contains only 4000 stars of $[\mathrm{Fe} / \mathrm{H}]<-1.0 \mathrm{dex}$, and no stars of $[\mathrm{Fe} / \mathrm{H}]>0.5 \mathrm{dex}$. We use summation in replacement of the integration in actual calculation.

Fig. 13 shows a color-coded distribution of the mean metallicities in the $R-Z$ plane. The left and right panels show respectively the results derived before and after applying the CMD weights deduced in Section 4.1. When plotting the distributions, we have discarded bins containing less than 30 stars. The distributions before and after CMD weight corrections are quite similar, suggesting that the CMD corrections have only a marginal effects on the mean metallicities. A strong negative radial metallicity gra- 


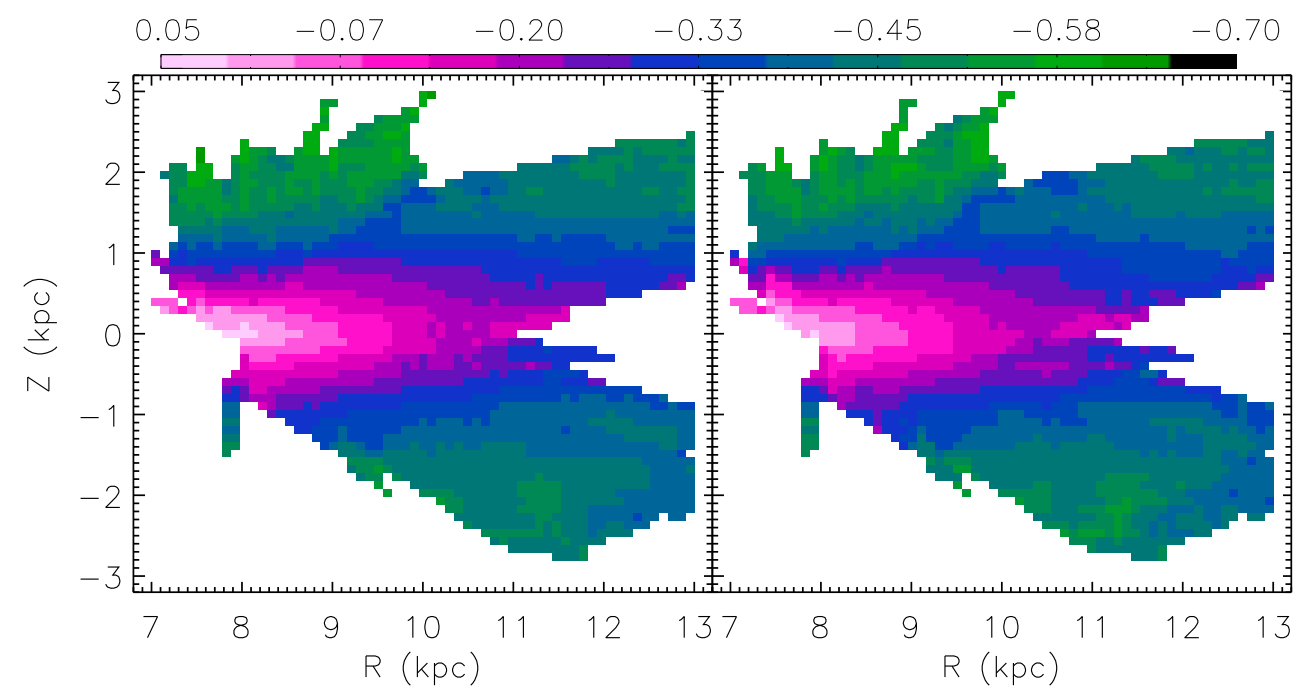

Fig. 13 Distributions of mean metallicities in the $R-Z$ plane before (left) and after (right) applying CMD weights for our sample stars.

dient is clearly seen near the disk plane, and the gradient flattens as one moves away from the midplane. There are also significant negative vertical gradients, which become shallower as one moves outward. The distributions are largely symmetric with respect to the disk plane. To quantitatively characterize the spatial and temporal variations of mean metallicities, we derive the radial and vertical metallicity gradients and study their temporal variations in the remaining part of this Section.

\subsection{Radial metallicity gradient}

\subsubsection{The radial gradients as a function of $|Z|$}

We divide the stars into slices of $|Z|$ of thickness of $0.2 \mathrm{kpc}$. In each slice, the mean metallicities of the individual bins in radial direction as a function of $R$ are fitted with a linear function. The slope of the fit is adopted as the radial metallicity gradient. In each slice, we divide the stars into radial bins by requiring that each radial bin contains a minimum of 100 stars. The minimum bin size is set to be $0.2 \mathrm{kpc}$. For stars in each radial bin, we calculate the mean metallicity and uncertainty, after applying the CMD weights to the individual stars, as well as the error-weighted mean and error of $R$. A linear function is then used to fit the mean metallicities as a function of $R$ to derive the radial metallicity gradient. Errors of the mean metallicities and the mean values of $R$ are taken into account in the fitting to estimate the error of the derived radial metallicity gradient.

In doing so, we divide the MSTO sample stars into groups of different age bins. Considering that the metallicity of the very young stars are probably biased against metal-poor ones due to an upper limit cut in $T_{\text {eff }}$ at $7500 \mathrm{~K}$ (cf. Fig. 2), we discard stars younger than $2 \mathrm{Gyr}$ to avoid the potential bias. The remaining stars are binned into groups of ages: $2-4 \mathrm{Gyr}, 4-6 \mathrm{Gyr}, 6-8 \mathrm{Gyr}, 8-11 \mathrm{Gyr}$ and $11-16$ Gyr. For each age group, we derive the radial metallicity gradients in different $|Z|$ slices. Fig. 14 shows the results for three $|Z|$ slices, $|Z|<0.1 \mathrm{kpc}, 0.4<|Z|<0.6 \mathrm{kpc}$ and $0.9<|Z|<1.1 \mathrm{kpc}$. The Figure shows that within a given $|Z|$ slice, stars of older ages are more metal-poor and exhibit shallower radial metallicity gradients.

The derived radial metallicity gradients as a function of $|Z|$ are plotted in Fig. 15. The Figure shows that the radial gradients have significant spatial and temporal variations. The gradients derived from 

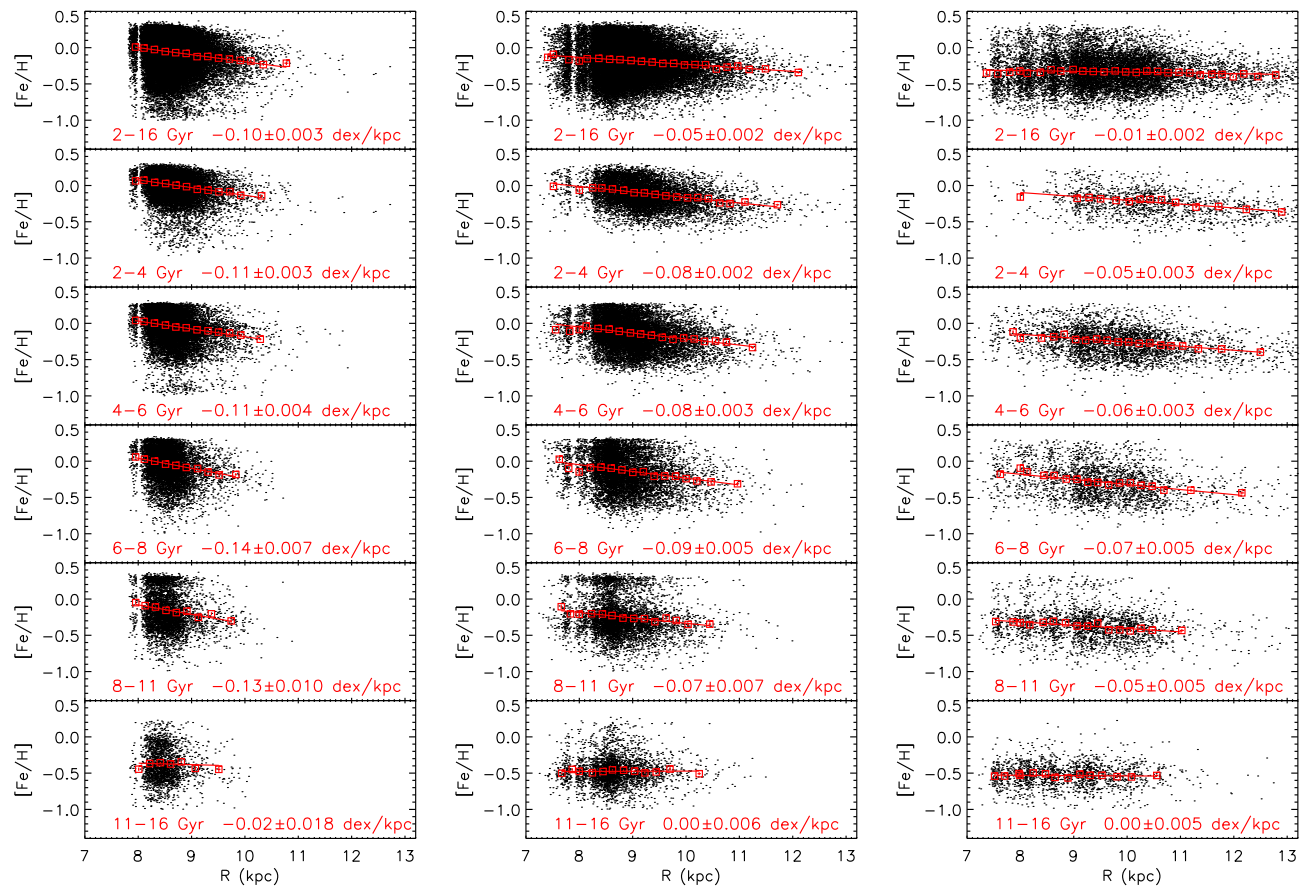

Fig. 14 Radial metallicity gradients $\Delta[\mathrm{Fe} / \mathrm{H}] / \Delta R$ derived from stars of $|Z|<0.1 \mathrm{kpc}$ (left panels), $0.4<|Z|<0.6 \mathrm{kpc}$ (middle panels) and $0.9<|Z|<1.1 \mathrm{kpc}$ (right panles) and of different age bins, as marked in each panel. Red squares represent mean metallicities in the individual radial bins, after applying the CMD weights. The error bars of the mean metallicities are overplotted, but in all cases they are smaller than the size of the symbols given the large number of stars in each radial bin. Red lines are linear fits to the red squares. The fitted gradient and associated error thus derived are marked in each panel.

stars of all ages (2-16 Gyr) increase monotonously, from $-0.1 \mathrm{dex} \mathrm{kpc}^{-1}$ at $|Z|=0 \mathrm{kpc}$ to $0.02 \mathrm{dex} \mathrm{kpc}^{-1}$ at $|Z|=2 \mathrm{kpc}$. However, stars of different age bins present significantly different gradients. In particular, stars of the oldest ages show essentially zero gradients at all heights, while stars of younger ages have negative gradients and the gradients from individual age bins are always steeper than those derived from stars of all ages. Except for those of the oldest age bin, gradients derived from stars of the individual age bins show significant spatial and moderate temporal variations: the gradients flatten with increasing height above the plane, and, as the lookback time decreases, the gradients first steepen, reaching a maximum (negative) value around $6-8 \mathrm{Gyr}$, and then become shallower again. The gradients derived from stars of some age bins (e.g. those of 6-8 Gyr and 8-11 Gyr) also show some fine features as $|Z|$ varies. The genuineness of those fine features is hard to assess for the moment given the potential selection effects of the current sample which has limiting magnitudes that vary from one sight line to another (cf. Section 4.2).

\subsubsection{The radial gradients as a function of age}

Fig. 16 plots the radial metallicity gradients as a function of age for stars in different $|Z|$ slices. It shows that at the earliest epochs (age $>8 \mathrm{Gyr}$ ), the gradients steepen with decreasing age, reaching a maximum around $6-8 \mathrm{Gyr}$, then flatten again. Similar trends are seen in all height slices of $|Z|<1.5 \mathrm{kpc}$. As 


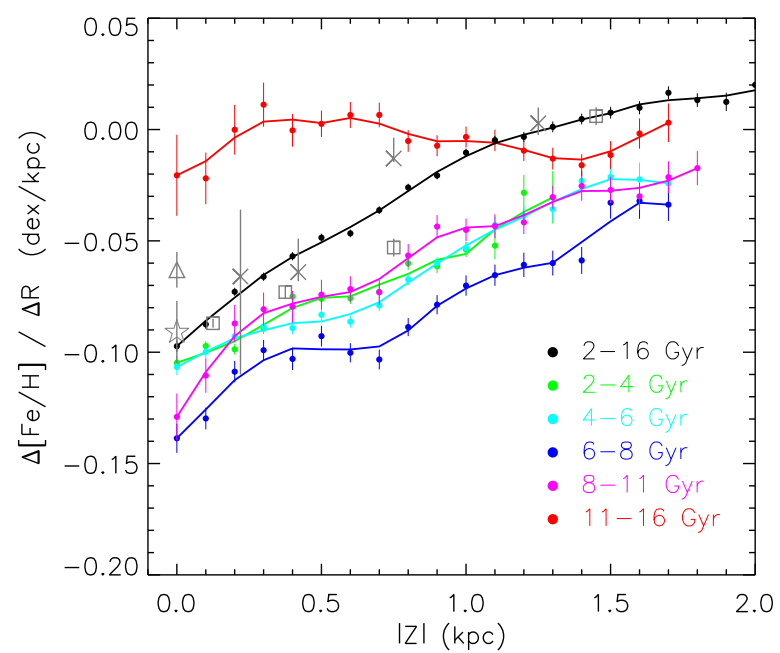

Fig. 15 Radial metallicity gradients as a function of $|Z|$ derived from stars of different age bins. Dots represent actual measurements, while lines are drawn by smoothing across 3 adjacent dots. Different colors represent results from stars of different age bins, as marked in the plot. Grey symbols are measurements from the literature. The triangle represents the measurement of Chen et al. (2003) using open clusters as tracers, the star is the measurement of Friel (1995), again using open clusters, the crosses are measurements of Cheng et al. (2012b) derived from SEGUE turn-off stars, and the squares are from Hayden et al. (2014) deduced from APOGEE giants.

already shown by Fig. 15, the negative gradients of stars younger than 11 Gyr flatten as $|Z|$ increases, while the gradients of the oldest $(>11 \mathrm{Gyr}$ ) stars are essentially zero and invariant with $|Z|$. As a result, stars at large $|Z|$ show only a weak trend of variations with age at the early epochs. For the height slice of $0.9<|Z|<1.1 \mathrm{kpc}$, the gradient steepens from a value of $0.0 \mathrm{dex} \mathrm{kpc}^{-1}$ around $12.5 \mathrm{Gyr}$ to $-0.07 \mathrm{dex} \mathrm{kpc}^{-1}$ at $7 \mathrm{Gyr}$, corresponding an evolution rate of $-0.013 \mathrm{dex} \mathrm{kpc}^{-1} \mathrm{Gyr}^{-1}$. The corresponding rate is $-0.020 \mathrm{dex} \mathrm{kpc}^{-1} \mathrm{Gyr}^{-1}$ for height slice $0.3<|Z|<0.5 \mathrm{kpc}$ and $-0.022 \mathrm{dex} \mathrm{kpc}^{-1} \mathrm{Gyr}^{-1} \mathrm{for}$ $|Z|<0.1 \mathrm{kpc}$. Analyses show that the radial gradients derived from stars of $7-9$ Gyr are comparable with those from stars of $6-8 \mathrm{Gyr}$, indicating that the gradients probably reach the maximum earlier, probably $8 \mathrm{Gyr}$ ago. This suggests that the evolution rate of the radial gradient at early epochs of the disk formation is even faster. Unfortunately, given the relatively large uncertainties and poor (absolute) calibration of our current age determinations, it is difficult to pin down the exact time that the radial gradients reach the maximum and the exact values of the evolution rate of the gradients. For stars of ages younger than $8 \mathrm{Gyr}$, as the age decreases, the gradients flatten and the evolution rate slows down to about $0.007 \mathrm{dex} \mathrm{kpc}^{-1} \mathrm{Gyr}^{-1}$, much slower than the rates at the early epochs for bins of low $|Z|$. The application of CMD weights to the data mainly changes the gradients in slices of lower $|Z|(<0.5 \mathrm{kpc})$, and leads to an increase of gradients between 0.01 and $0.03 \mathrm{dex} \mathrm{kpc}^{-1}$. Nevertheless, the CMD correction does not change the overall trend of gradients as a function of age.

\subsubsection{Comparison with previous results}

In Fig. 15 we have also plotted measurements of the radial gradients from several recent studies, including the measurement of Cheng et al. (2012b) using SEGUE F turn-off stars, that of Hayden et al. (2014) deduced from APOGEE red giants, and those of Friel (1995) and Chen et al. (2003) using open clusters 

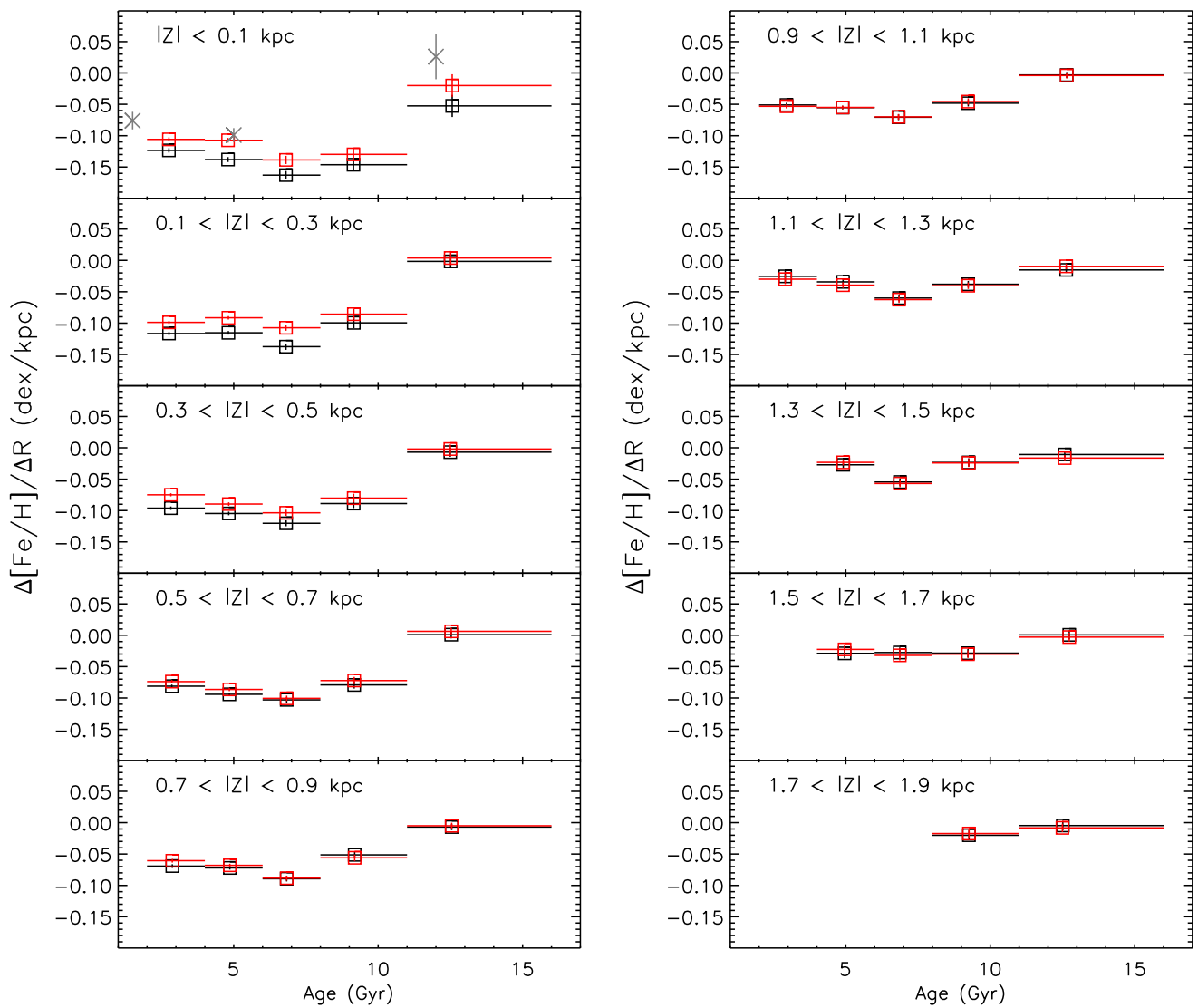

Fig. 16 Radial metallicity gradients as a function of age for stars in different $|Z|$ bins, as marked in the plot. The black and red squares represent respectively results before and after applying the CMD weights to our sample stars. The horizontal error bars represent the age ranges of stars adopted to derive the radial gradients. The vertical error bars are the fitting errors of radial gradients, and in most cases they are very small because of the large number of stars. They grey crosses in the $|Z|<0.1 \mathrm{kpc}$ bin are measurements from Nordström et al. (2004).

as tracers. The Figure shows that the gradients and their variations with $|Z|$ given by those earlier studies are largely consistent with our results deduced from stars of all ages, except for the bin of $|Z|=0.75 \mathrm{kpc}$

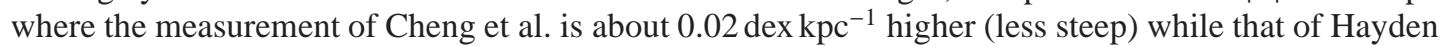
et al. is lower by a similar amount (more steep). At $|Z| \sim 0.1 \mathrm{kpc}$, the gradient, $-0.063 \pm 0.008 \mathrm{dex} \mathrm{kpc}^{-1}$, derived from an analysis of 118 open clusters by Chen et al. (2003), seems to be too high (less steep), by as much as $0.03 \mathrm{dex} \mathrm{kpc}^{-1}$, compared to our result as well as that of Friel (1995), who find a gradient of $-0.091 \pm 0.014 \mathrm{dex} \mathrm{kpc}^{-1}$ using 44 open clusters between 7 and $16 \mathrm{kpc}$.

We show in Section 4 that metallicity gradients derived from stars of a wide range of ages suffer from strong selection effects. In general the effects lead to shallower (negative) metallicity gradient than the true value (cf. Section 4.2), or even a positive gradient at large $|Z|$. Both our gradients derived from stars of all ages and those of Cheng et al. are likely suffered from such effects. On the other hand, as we show in Section 4, our gradients deduced from stars of the individual age bins should be hardly affected 
by those effects. Hayden et al. (2014) divide their sample into stars of high- $[\alpha / \mathrm{Fe}]$, which they believe to be mainly consist of old stars, and stars of low- $[\alpha / \mathrm{Fe}]$, which they believe to be mostly young stars. For the young stellar population, they find a radial metallicity gradient of $-0.03 \pm 0.006 \mathrm{dex} \mathrm{kpc}^{-1}$ for stars of heights $1.0<|Z|<2.0 \mathrm{kpc}$. This value is basically in agreement with our results for stars younger than $11 \mathrm{Gyr}$ in the same height range. For the old, high- $[\alpha / \mathrm{Fe}]$ population, Hayden et al. find that the radial metallicity gradients show significant variations with $|Z|$, in contrast to our results. For stars of the oldest ages $(>11 \mathrm{Gyr})$, we find that the radial gradients are essentially zero at all heights covered by the current sample $(<2 \mathrm{kpc})$. Most open clusters have ages younger than $2 \mathrm{Gyr}$, and they generally yield a negative radial gradient ranging, for example, from $-0.09 \mathrm{dex} \mathrm{kpc}^{-1}$ (Friel \& Janes 1993; Friel 1995) to $-0.06 \mathrm{dex} \mathrm{kpc}^{-1}$ (Friel et al. 2002; Chen et al. 2003). Parts of the scatter of results may be caused by the different ranges of $R$ and $Z$, maybe also of age covered by the different samples of open clusters employed in the individual studies. As described earlier (Section 5.2.1), to avoid bias, we have excluded stars younger than $2 \mathrm{Gyr}$ in our sample. Our results deduced from stars older than $2 \mathrm{Gyr}$ show that the gradients flatten as age decreasing in the past few Gyr. Thus the relatively shallower gradients yielded by open clusters compared to our measurements from young stars near the disk plane is probably not surprising. Note also that the sample employed by Chen et al. (2003) includes open clusters spreading over a wide range of age and $|Z|$. This may have also caused the relatively shallower radial gradient found by them.

In Fig. 16 we have also plotted the measurements of Nordström et al. (2004) in the panel of $|Z|<$ $0.1 \mathrm{kpc}$ (top-left), considering that their stars are limited to a small volume of the solar neighborhood. Note that Nordström et al. use the mean orbital radii rather than the measured values of $R$ in deriving the gradients. The overall trend of variations of gradients with age deduced from this very local sample of stars is in good agreement with that reported here, although there are some differences in their absolute values. Nordström et al. (2004) suggest that the gradients of young disk stars steepen mildly with age, but stars of the oldest ages ( $>10 \mathrm{Gyr}$ ) do not follow the trend. In fact, results from PNe also suggest that the radial metallicity gradients of the Galactic disk steepen with age (Maciel et al., 2003; Maciel \& Costa, 2009). All these are consistent with what we see in the current data, in much more detail.

\subsection{Vertical metallicity gradients}

\subsubsection{Vertical gradients as a function of $R$ and age}

To derive the metallicity gradients along the vertical direction, we divide the stars into annuli of $1 \mathrm{kpc}$ in the radial direction. In each annulus, we further divide the stars into bins of $|Z|$ by requiring that each vertical bin contains at least 100 stars that cover at least $0.2 \mathrm{kpc}$ in the vertical direction. The slope of a linear fit to the CMD weight corrected mean metallicities as a function of $|Z|$ is adopted as the vertical gradient for that radial annulus. As in the case for radial gradients, we divide the sample stars into groups of different age bins to examine the temporal variations of vertical gradients.

Fig. 17 plots the vertical gradients derived from stars of different age bins as a function of the mean radius $R$ of the radial annulus. It shows that for stars of almost all age bins, the vertical gradients flatten as $R$ increases. The only exception are those derived from the oldest ( $>11 \mathrm{Gyr}$ ) stars - the vertical gradients yielded by those old stars are largely constant, at the level of about $-0.11 \mathrm{dex} \mathrm{kpc}^{-1}$, becoming only slightly shallower by about $0.02 \mathrm{dex} \mathrm{kpc}^{-1}$ from 8 to $11 \mathrm{kpc}$. In fact, considering the uncertainties of the gradients and the systematics induced by potential unaccounted for selection effects the vertical gradients derived from the oldest stars are essentially consistent with no radial evolution at all. Phenomenologically, it is probably not surprising that one sees such trends of variations of vertical gradients as a function of $R$ given that we have already shown that, except for those of oldest ages, our sample stars exhibit negative values of radial metallicity gradients that become shallower as $|Z|$ increases, while for those oldest stars the radial gradients are essentially zero at all heights (cf. Figs. 15, 16).

Fig. 17 also shows that the vertical gradients derived from stars of all ages are significantly steeper than those derived from stars of the individual age bins. This result reflects the differences of the spatial 


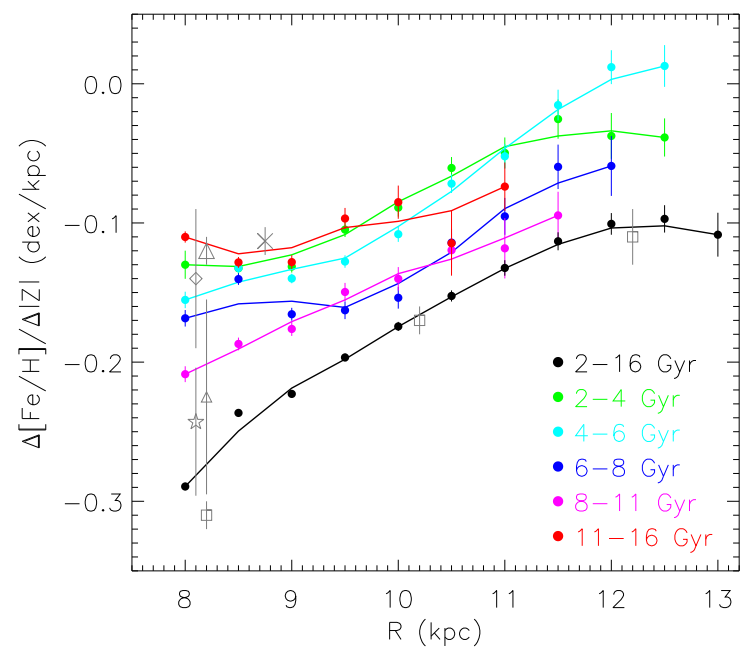

Fig. 17 Same as Fig. 15 but for vertical gradients as a function of $R$. The grey symbols are measurements from APOGEE giants (squares) by Hayden et al. (2014), SEGUE stars (five pointed star) by Schlesinger et al. (2014), SEGUE FGK dwarfs of $1<|Z|<3 \mathrm{kpc}$ (cross) by Carrell et al. (2012), thick disk stars of $1<|Z|<4 \mathrm{kpc}$ (diamond) by Kordopatis et al. (2011), SEGUE RHB stars of $|Z|<3 \mathrm{kpc}$ (small triangle), and from thick disk RHB stars of $0.5<|Z|<3 \mathrm{kpc}$ (large triangle) by Chen et al. (2011).

distribution of stars of different ages. Young stars, mostly metal-rich, concentrate at small heights, while those of older ages, often more metal-poor stars, are more often found at larger heights. This leads to a steeper negative vertical gradients if stars of all ages are put together to derive the gradients. Note that this is different from the case for the radial gradients, where if one includes stars of all ages when determining the metallicity gradients, then the selection effects will lead to shallower gradients than the true underlying values.

At a given $R$, the vertical gradients show significant evolution with ages. The oldest stars have a nearly constant vertical gradient of about $-0.11 \pm 0.02 \mathrm{dex} \mathrm{kpc}^{-1}$. The gradients become much steeper at age bins $8-11 \mathrm{Gyr}$ and $6-8 \mathrm{Gyr}$, and then become shallower again. This is seen in all annuli of $R<11 \mathrm{kpc}$. In the radial annulus of $R=9.0 \mathrm{kpc}$, where the sample suffers from the least selection bias as a result of the magnitude cuts both at the faint and the bright ends, it seems that $8 \mathrm{Gyr}$ is an apparent turning point of the vertical gradients. It is difficult to assess at the moment whether the epoch of this turning point of vertical gradients varies with $R$ or not due to the potential systematics of our current measurements at both small and large radii. However, the presence of a turning epoch around $8 \mathrm{Gyr}$, with the exact time that may differ by one or two Gyr, seems to be secure. At $R=9.0 \mathrm{kpc}$, the vertical gradients steepen at a rate of about $0.01 \mathrm{dex} \mathrm{kpc}^{-1} \mathrm{Gyr}^{-1}$ from the earliest epoch $(\sim 12.5 \mathrm{Gyr})$ to $8 \mathrm{Gyr}$, and then flatten with a comparable speed at later epochs.

\subsubsection{Comparison with previous results}

In Fig. 17, we also show some recent measurements of the vertical gradients, including those of Chen et al. (2011) utilizing SEGUE red horizontal branch (RHB) stars, Kordopatis et al. (2011) utilizing thick disk stars in the solar neighborhood, Carrell et al. (2012) and Schlesinger et al. (2014) utilizing SEGUE FGK stars, as well as Hayden et al. (2014) utilizing APOGEE red giants. Note that since in many of those studies the exact mean radial position of sample stars in not given, we have inferred a rough 
value based on the published plots. The comparison shows that the values of vertical gradients derived from the current sample of MSTO stars of all ages are well consistent with determinations of Hayden et al. (2014), who find a value of about $-0.31,-0.17$ and $-0.11 \mathrm{dex} \mathrm{kpc}^{-1}$ at $R=8,10$ and $12 \mathrm{kpc}$, respectively. From a sample of RHB stars of $|Z|<3.0 \mathrm{kpc}$, Chen et al. (2011) find a vertical gradient of $-0.225 \mathrm{dex} \mathrm{kpc}^{-1}$, comparable to that found by Schlesinger et al. (2014) and consistent with our result found from stars of all ages if one takes into account the relative large uncertainties of measurement of Chen et al. Note that after excluding the thin disk and halo stars, Chen et al. find a vertical gradient of $-0.12 \mathrm{dex} \mathrm{kpc}^{-1}$ from the remaining thick disk stars of $0.5<|Z|<3 \mathrm{kpc}$. This result is corroborated by Schlesinger et al. based on a similar exercise. Similarly, Carrell et al. (2012) find a vertical gradient of $-0.113 \mathrm{dex} \mathrm{kpc}^{-1}$ from SEGUE FGK dwarfs of $1<|Z|<3 \mathrm{kpc}$, where the thick disk stars are expected to dominate. Here for stars of the oldest ages, we find a gradient of $-0.12 \mathrm{dex} \mathrm{kpc}^{-1}$. All these results seem to support our conclusion that the vertical gradients show significant temporal variations. They also support our conjecture that those stars of oldest ages in our sample are actually thick disk stars (cf. Section 6).

\section{DISCUSSION AND IMPLICATION}

In this section, we discuss the uncertainties of our results, as well as the implication of our findings of metallicity gradients on the nature of the thick disk and how that may constrain the formation and evolution history of the thin and thick disks.

\subsection{Uncertainties of measurements}

There are two main error sources that introduce uncertainties to our determinations of metallicity gradients, the errors of stellar atmospheric parameters and the potential selection effects hidden in a magnitude limited sample.

Errors in the stellar atmospheric parameters lead to uncertainties in the estimated distances and ages, and introduce contamination, say by dwarfs, in our MSTO star sample. The largest uncertainties are probably come from $\log g$ estimates. The LSP3 values of $\log g$ adopted in the current work suffers from the boundary effects (Xiang et al. 2015b). Some moderate systematic errors are also found to exist in the adopted $\log g$ via a comparison with the asteroseismic values (Ren et al. 2015, in prep.). These systematic errors have not been corrected for in the current work. To account for the potential systematics in distance estimates induced by errors in the stellar atmospheric parameters, we have calibrated the distances derived using the Hipparcos distance measurements of the MILES spectral template stars. The solution works well for MSTO stars, which constitute the majority of MILES stars in the temperature range $5400<T_{\text {eff }}<7500 \mathrm{~K}$. However, there are some contaminations in our MSTO star sample from dwarf or subgiant stars as a result of the boundary effects for $\log g$ determinations. For those contaminating stars, their distances are either over- or underestimated, and their ages are always overestimated. Such effects mainly occur near the low temperature cut, where stellar isochrones of different ages are closely packed in the $T_{\text {eff }}-\log g$ diagram (Fig. 1). Near the low temperature end $\left(T_{\text {eff }}<5500 \mathrm{~K}\right)$, there are also some weak clumping of metal-rich stars in the $T_{\text {eff }}-[\mathrm{Fe} / \mathrm{H}]$ plane (e.g. Fig. 42 of Yuan et al. 2015a), which are possibly artifacts in the LSP3 parameters, caused by the clustering and holes in the distributions of MILES template stars in the parameter space. The clumping of metal-rich stars seen in the $8-11 \mathrm{Gyr}$ age bins of Fig. 14 are in fact contributed by those stars. A comparison with the LAMOST DR1 parameters show that those stars are also metal-rich $([\mathrm{Fe} / \mathrm{H}]>0$ dex $)$ in the LAMOST DR1, though their distribution is smoother in the space of LAMOST DR1 parameters than in that of LSS-GAC parameters.

To further investigate that how our results may have been affected by distance errors, we reassign distances to the MSTO sample stars based on their distance errors using a Monte Carlo technique and redo the analysis presented in Section 5. We have performed 500 simulations. In most cases, the resultant gradients as well as the trends of their spatial and temporal variations show no significant changes compared with those presented in Section 5, suggesting that our results are not significantly affected by 
the distance errors. The only noticeable change is that some of the fine features seen in the trends of radial gradients as a function $|Z|$ (Fig. 15) disappear in some of the simulations, suggesting that those fine features are probably artifacts caused, at least in parts, by the distance uncertainties.

We have implemented several tests to examine the potential effects of contamination induced by dwarfs and subgiants in our sample. First, we continuously shrink the $T_{\text {eff }}$ range of our MSTO star sample to reduce the level of contamination, and then redo the analysis. We find that even with a very restrict range of $5700<T_{\text {eff }}<6500 \mathrm{~K}$, the data still yield results basically consistent with those presented in the current work. This implies that contamination from dwarfs and subgiants probably does not have a big impact on our study. There, however, are some noticeable changes in the results of radial gradients at $|Z|<0.4 \mathrm{kpc}$ deduced from stars older than $6 \mathrm{Gyr}$, as they become flatter. Those changes are understandable. With a $T_{\text {eff }}$ cut of $5700 \mathrm{~K}$, the sample become significantly biased in the sense that old, metal-rich stars have been systematically excluded from the sample, leading to an artificially flatter radial gradients. Nevertheless, the changes do not affect our main conclusion that the metallicity gradients show significant spatial and temporal variations, and that the radial metallicity gradients of the oldest stars are essentially zero at all heights. Our other tests include repeating the current analysis using a MSTO star sample selected from the official LAMOST DR2, as well as using new LSP3 parameters estimated with a KPCA method, which will be implemented in the next release of LSP3 parameters, to be included in the value-added catalog of LSS-GAC DR2. We find that measurements of stellar atmospheric parameters yielded by different pipelines give results consistent with what we presented in the current work. We thus believe that uncertainties in stellar atmospheric parameters adopted in the current work do not affect our results significantly. More accurate parameters will however be very useful to characterize the temporal variations of metallicity gradients, both in radial and vertical directions, in particular to pin down the exact turning epoch when the evolution of gradients changes from a mode of steeping with decreasing age to a mode of flattening with decreasing age, as well as the exact epochs when the thick disk formation started and ceased.

As discussed in Section 4, our sample is a magnitude limited one. The sample is biased against metal-poor stars near the farther distance limit corresponding to magnitude cut at the faint end and biased in favour of more metal-poor stars near the nearer distance end corresponding to the magnitude cut at the bright end. The magnitudes of those effects vary from one sight line to another. The effects may be responsible for some of the fine features seen in Figs. 15-17. We expect that as the survey progresses, the sky coverage of the VB, B and M plates continues to improve, such effects will be much less important.

\subsection{Thin and thick disks - two different phases of the disk formation?}

We show that sample stars of the oldest ages ( $>11 \mathrm{Gyr}$ ) have essentially zero radial metallicity gradients at all heights, completely different to the behaviour of stars of younger ages. The latter exhibit negative radial gradients that flatten with increasing height. In the direction perpendicular to the disk, stars of oldest ages show vertical gradients almost invariant with $R$, while stars of younger ages have gradients that flatten significantly with increasing $R$. These results strongly suggest that the stars of the oldest ages have experienced a formation process very different to stars of younger ages. It seems that those oldest disk stars formed from gas that is well-mixed in the radial direction, while younger stars formed from radially inadequately mixed gas. It is widely accepted that gas accretion and inflow play a key role in providing gas and determining the star formation history of the Galactic disks. Gas inflow is expected to transfer metals inwards, increasing the radial (negative) metallicity gradients of the disk (e.g. Lacey \& Fall 1985; Schönrich \& Binney 2009a). This suggests that gas accretion and inflow that determine the star formation and enrichment history of the younger disk did not play a significant role in the formation of the old disk. Rather the old disk seems to have been formed via some violent, fast processes, such as gas collapse or merging.

The existence of a thick disk (Gilmore \& Reid 1983) of the Milky Way was first proposed more than 30 years. Over the years, much have been learned with regard to the properties of the thick disk and various criteria have been proposed to characterize the thick disk stars, such as the spatial position 
in the vertical direction $|Z|$, the 3-dimensional velocity with respect to the local standard of rest (LSR) and the metallicity $[\mathrm{Fe} / \mathrm{H}]$ and $\alpha$-element to iron abundance ratio $[\alpha / \mathrm{Fe}]$. To distinguish thick disk stars from thin disk stars based on their spatial positions alone is difficult. At low Galactic latitudes, the disk is dominated by thin disk stars. Even at relatively high latitudes, the contaminations of thin disk stars could be substantial. The kinematics of disk stars are affected by a variety of processes (churning and blurring) related with asymmetric (sub)structures (the bar, spirals, and stellar streams). Samples of thick disk stars selected purely based on stellar kinematics are also often biased in metallicity since there seems to be a correlation between the velocity and metallicity of a disk star due to secular evolution of the Galactic disk (e.g. Lee et al. 2011). As for metallicity, there seems to be no physical base to give a clear cut that distinguish the thin and thick disk stars. In the current, we find that stars of the oldest ages are more metal-poor and exhibit vertical gradients in excellent agreement with the results derived from thick disk stars in previous studies. More importantly, it seems that stars of the oldest ages have radial metallicity gradients comparable to those yielded by stars of all ages at large disk heights, where the thick disk stars are expected to dominate. In a parallel work, we show that the mean age of stars increase with disk heights, and stars of the oldest ages dominate the populations at large heights (Xiang et al. in prep.). This suggests that disk stars of the oldest ages are in fact thick disk stars. We thus propose that age is a natural, clear criterion that distinguishes the thin and thick disk stars: stars of the oldest ages belong to the thick disk while those of younger ages are from the thin disk.

The above discussion suggests that the thick disk corresponds an earlier phase of the disk formation via processes that seem to be quite different from those responsible for the formation of the younger, thin disk. This is in conflict with the recent suggestion of Bovy et al. (2012), who suggest that the thin and thick disks are probably not two distinct components of the disk in terms of origin based on their finding that in the $[\mathrm{Fe} / \mathrm{H}]-[\alpha / \mathrm{Fe}]$ plane, stellar populations of the largest scale heights have the smallest scale lengths. On the other hand, Haywood et al. (2013) have recently studied the age $-[\mathrm{Fe} / \mathrm{H}]$ and age $-[\alpha / \mathrm{Fe}]$ relations of 1111 FGK stars in the solar neighborhood. They find that thick disk stars show a tight correlation of $[\alpha / \mathrm{Fe}]$ and $[\mathrm{Fe} / \mathrm{H}]$ with age. They thus suggest that thick disk stars have an origin different to that of the younger, thin disk stars. Haywood et al. (2013) suggest that the older, thick disk formed from well-mixed interstellar medium over a time scale of $4-5 \mathrm{Gyr}$ at $8 \mathrm{Gyr}$ ago, the inner thin disk formed later, with the initial chemical conditions set by the youngest thick disk. While the outer thin disk is different, as it has began to form at $10 \mathrm{Gyr}$ ago. Our results suggest the idea that the gas is probably well-mixed in the radial direction $11 \mathrm{Gyr}$ ago, but probably not so in the vertical direction. Stars of age between 8 and 11 Gyr begin to show some radial gradients, and they probably a mixture of thin and thick disk stars. Given the relatively large uncertainties of our age estimates, the exact epoch when the thick disk formation ceased and the thin disk formation prevailed remains to be determined. The epoch should be sometime around $11 \mathrm{Gyr}$ but in any case earlier than $8 \mathrm{Gyr}$. The transition must be rapid since we see an abrupt change in the slopes of both radial and vertical metallicity gradients between stars of ages older than $11 \mathrm{Gyr}$ and those of ages between 8 and $11 \mathrm{Gyr}$. Thus, we suspect that thick disk may have formed slightly quicker than claimed by Haywood et al. With regard to the thin disk, there is no strong evidence to group them into those from the inner and those from the outer parts of the thin disk in discussing their possible formation scenarios.

\subsection{Constraining the disk formation scenarios}

With regard to the thick disk formation, as discussed above, our results prefer a violent, fast formation process. One possibility is the scenario of a fast, highly-turbulent gas-rich merger formation as proposed by Brook et al. $(2004,2005)$. The scenario seems to be preferred by a number of authors in explaining their results of metallicity gradients (e.g. Cheng et al. 2012b; Schlesinger et al. 2012, 2014; Kordopatis et al. 2011; Boche et al. 2013; Hayden et al. 2014). Nevertheless, we note that based on their simulations, Brook et al. suggest that the thick disk formation occurred so fast $\sim 12$ Gyr ago that there should be no metallicity gradients at all in both the radial and vertical directions. However, On the other hand, significant vertical gradients for thick disk stars have been detected by many studies, including the current one. This may imply that the thick disk formation process of Brook et al. is probably too fast 
and violent. In fact, Gilmore et al. (1989) point out that a slow, pressure-supported gas collapse following the formation of extreme Population II system could form a thick disk. Based on the hydrodynamical models of Larson (1976), disk formed in such a way shows weak radial metallicity gradients in the solar neighborhood but strong vertical gradients because the vertical flow dominates when the gas settles down to disk at that epoch. Such a slow, pressure-supported collapse formation scenario seems to be consistent with our results, though better, quantitive models/simulations are needed to demonstrate this.

It has also been proposed that the thick disk is produced by stellar radial migration (e.g. Roškar et al. 2008; Schönrich \& Binney 2009b; Loebman et al. 2011). Our results show that at $|Z|<1.0 \mathrm{kpc}$, the radial gradients of the old, thick disk stars, as well as their trend of variations with $|Z|$, are significantly different from those derived from stars of age bins $8-11 \mathrm{Gyr}$ and $6-8 \mathrm{Gyr}$. In addition, for a given $|Z|$, there is a turning in the temporal variations of radial gradients, at an epoch of $\sim 8 \mathrm{Gyr}$, after which the gradients become flatter as age decreases. These results are difficult to explain by radial migration since radial migration is expected to reduce the radial gradients (e.g. Loebman et al. 2011; Kubryk et al. 2013), and as such one expects a continuous rather than abrupt temporal variations. Note that this does not imply there is no radial migration, but that the radial migration is probably not the dominant mechanism that shape the properties of metallicity gradients of concern. The observed metallicity gradients are likely the results of both gas infall/inflow, which steepens the gradients, and stellar migration, which flattens the gradients. Based on results presented in the current work, it seems to us that gas infall/inflow probably dominates in shaping the metallicity gradients of the disk and their temporal variations. In fact, there is simulations suggesting that stellar radial migration does not change the trend of variations of radial metallicity gradients (Curir et al. 2014).

For the thin disk evolution, it has been suggested that stellar radial migration has played an important role considering that it successfully explain the age - metallicity distribution and metallicity - velocity relations of stars in the solar neighborhood (e.g. Schönrich \& Binney 2009b; Lee et al. 2011; Loebman et al. 2011). Our current analyses show that the radial gradients deduced from young, thin disk stars flatten with increasing $|Z|$. Similarly, the vertical gradients flatten as $R$ increases. These trends are probably the consequence of outward stellar radial migration, since as a star migrates outward, its altitude is expected to increase significantly (e.g. Minchev et al. 2012). In addition, both metal-rich stars of very old ages (e.g. $8-11 \mathrm{Gyr}$ ), and metal-poor stars of very young ages (e.g. < $4 \mathrm{Gyr}$ ) are present in the solar neighborhood (Fig. 14). A direct examination of the age - metallicity distribution shows similar results. This is clear signature of stellar radial migration, which brings inner, metal-rich stars outward, and outer, metal-poor stars inward. In the panels of Fig. 14 showing the results of stars of the oldest ages, there are very few old metal-rich stars compared to panels showing the results of stars of younger age bins. This again suggests that the thick disk does not result from radial migration of thin disk stars. It seems that $8 \mathrm{Gyr}$ is a specific epoch in the formation history of the thin disk, since it is around this epoch that both the radial and vertical metallicity gradients reach their maximum (negative) values. We suspect that around $8 \mathrm{Gyr}$, the gas accretion/inflow rate in the solar neighbourhood reached the maximum.

\section{SUMMARY}

In this work, we have defined a sample of 297042 MSTO stars of effective temperature range of $5400<$ $T_{\text {eff }}<7500 \mathrm{~K}$ from the LSS-GAC survey. Most of the sample objects are disk stars of metallicity $[\mathrm{Fe} / \mathrm{H}]>-1.0 \mathrm{dex}$. The sample is used to study the metallicity gradients of the Galactic disk in the anti-center direction. For any age between 2 and $13 \mathrm{Gyr}$, our sample encompasses stars spreading over almost the whole metallicity range of typical disk stars. We estimate distances and ages of the sample stars by isochrone fitting, and achieve an accuracy of about 20 per cent in distance and 30 per cent in age. The sample covers a contiguous space volume from 7.5 to $13.5 \mathrm{kpc}$ in Galactocentric radial distance $R$ and from -2.5 to $2.5 \mathrm{kpc}$ in disk height $Z$.

To correct for the sample selection effects, we first apply a CMD weight to the individual sample star, leads us to a magnitude limited sample. We then characterize the potential biases of metallicity gradients deduced from a magnitude limited sample utilizing mock data generated with Monte Carlo simulations of the Galactic disk. We find that the radial metallicity gradients derived from a sample 
of stars in a narrow range of ages reproduce the underlying true values well. However, if the sample includes stars over a wide range of ages, then the radial gradients derived will be severely biased to larger values (i.e. less steep for negative radial gradients which is the case for the real Galactic disk).

Both the radial and the vertical metallicity gradients are found to show significant spatial and temporal variations. The radial gradients, $\Delta[\mathrm{Fe} / \mathrm{H}] / \Delta R$, deduced from stars of the oldest ages $(>11 \mathrm{Gyr})$ are essentially zero at all heights above the Galactic disk plane, while those deduced from stars in younger age bins are always negative at all heights. The vertical gradients, $\Delta[\mathrm{Fe} / \mathrm{H}] / \Delta|\mathrm{Z}|$, deduced from stars of the oldest ages are about $-0.1 \mathrm{dex} \mathrm{kpc}^{-1}$, with some marginal variations with $R$, while those deduced from stars in younger age bins show large variations with $R$. Based on the results, we infer that the Galactic disk formation may have experienced at least two distinct phases. In the earlier phase $(\sim 11 \mathrm{Gyr}$ ago), gas settled down to the disk mainly along the direction perpendicular to and produced stars with a negative vertical gradient. The gas is well-mixed in the radial direction such that the (thick disk) stars formed exhibit no radial gradients. In the later phase, the disk build up mainly by gas accretion/inflow in the disk, yielding negative radial gradients. Our metallicity gradients deduced from stars of the oldest ages are well consistent with the previous determinations using samples of thick disk stars selected via either spatial or chemical criteria (e.g. Chen et al. 2011; Carrell et al. 2012; Cheng et al. 2012b). We thus suggest that the thick component of the Galactic disk corresponds to an earlier phase of disk formation, while the thin component corresponds a later phase of disk buildup. We propose that stellar age serve as a natural, physical quantity to distinguish the thin and thick disk stars. Given that the thick disk shows negative vertical gradients, the gas-rich merger scenario of Brook et al. $(2004,2005)$ is not preferred, since in this scenario the thick disk forms so fast that it does not produce significant negative vertical gradient. On the other hand, a slow, pressure-supported collapse of gas following the formation of the Population II system, as first outlined by Gilmore et al. (1989), is more consistent with the current observations. There is an abrupt, significant change in both the radial and vertical metallicity gradients from the thick to the thin disk, suggesting that stellar radial migration (e.g. Schönrich \& Binney, 2009b; Loebman et al. 2011) might not play an important role in the thick disk formation.

At the earliest epochs, the radial gradients steepen with decreasing age, reaching maxima around $7-8 \mathrm{Gyr}$ ago, after which the gradients flatten. Such trends of temporal variations are seen at all disk heights below $1.5 \mathrm{kpc}$. Similar trends of variations are also observed in the vertical gradients. It seems that $8 \mathrm{Gyr}$ is a specific epoch in the buildup of the thin disk. It is possible that around this epoch, the gas accretion/inflow rate in the solar neighborhood reaches a maximum. This does not necessarily imply that the thin disk started to form only $8 \mathrm{Gyr}$ ago. There is likely a transition period, probably before $8 \mathrm{Gyr}$ ago, during which the disk buildup mode (in term of gas infall, accretion/inflow) changed from that of thick disk to the one of thin disk. Due to the relatively large uncertainties of our current age estimates, the exact epoch that this transition occurred remains to be determined. The fact that the radial metallicity gradients of the thin disk steepen with decreasing age implies that stellar radial migration is also unlikely to have played a decisive role in the formation and evolution of metallicity gradients of the thin disk.

A two-phase formation of the Galactic disk has been previously suggested by Haywood et al. (2013). The observations presented here however provide more details to this picture. For example, the presence of negative vertical metallicity gradients at the earliest epochs seem to suggest that gas infall at those earliest epochs are mainly along the direction perpendicular to the disk. The results also point to a slow, pressure-supported collapse of gas following the formation of the Population II system (Gilmore et al. 1989) for the thick disk formation. Our data also seem to suggest that the formation of thin disk started earlier than proposed by Haywood et al., probably sometime between 8 and 11 Gyr. Finally, Haywood et al. suggest that the inner and outer disks may have different origins, with the outer disk started to form earlier than the inner disk. They suggest that stars in the inner (metal-rich) thin disk are all younger than $8 \mathrm{Gyr}$, while in the outer (metal-poor) thin disk there are stars as old as $9-10 \mathrm{Gyr}$. However, our analysis seem to suggest that dividing the thin disk into the inner and outer parts in terms of different formation epochs is probably not necessary and substantiated by the data. 
Acknowledgments This work is supported by National Key Basic Research Program of China 2014CB845700. Guoshoujing Telescope (the Large Sky Area Multi-Object Fiber Spectroscopic Telescope LAMOST) is a National Major Scientific Project built by the Chinese Academy of Sciences. Funding for the project has been provided by the National Development and Reform Commission. LAMOST is operated and managed by the National Astronomical Observatories, Chinese Academy of Sciences. This work is also supported by National Natural Science Foundation of China (Grant No. 11473001). M.S.X. thanks Professor Jian-Ning Fu for providing the LAMOST data of Kepler fields. B.Q.C acknowledges partial funding from China Postdoctoral Science Foundation 2014M560843.

\section{References}

Abadi, M. G., Navarro, J. F., Steinmetz, M. \& Eke, V. R., 2003, ApJ, 597, 21

Anderson E. \& Francis Ch., 2012, AstL, 38, 331

Andrievsky, S. M., Kovtyukh, V. V. \& Luck, R. E. et al. 2002a, A\&A, 381, 32

Andrievsky, S. M., Luck, R. E., Martin, P. \& Lépine, J. R. D., 2004, A\&A, 413, 159

Balser, D. S., Rood, R. T., Bania, T. M. \& Anderson, L. D., 2011, ApJ, 738, 27

Bensby, T., Feltzing, S. \& Lundström, I. 2003, A\&A, 410, 527

Bensby, T., Feltzing, S., Lundström, I. \& Ilyin, I. 2005, A\&A, 433, 185

Bilir, S., Karaali, S., Ak, S. et al. 2012, MNRAS, 421, 3362

Bird, J. C., Kazantzidis, S. \& Weinberg, D. H., 2012, MNRAS, 420, 913

Boeche, C., Siebert, A., Piffl, T. et al. 2013, A\&A, 559, 59

Bournaud, F., Elmegreen, B. G. \& Martig, M., 2009, ApJ, 707, 1

Bovy, J., Rix, H. W., Liu, C. et al. 2012, ApJ, 753, 148

Brook, C. B., Kawata, D., Gibson, B. K. \& Freeman, K. C., 2004, ApJ, 612, 894

Brook, C. B., Gibson, B. K., Martel, H. \& Kawata, D., 2005, ApJ, 630, 298

Burstein, D. 1979, ApJ, 234, 829

Carpenter J. M., 2001, AJ, 121, 2851

Carrell, K., Chen, Y.-Q. \& Zhao, G. 2012, AJ, 144, 185

Chen, L., Hou, J. L. \& Wang, J. J., 2003, AJ, 125, 1397

Chen, Y. Q., Zhao, G., Zhao, J. K. et al. 2010, AJ, 140, 500

Chen, Y. Q., Zhao, G., Carrell, K., Zhao, J. K., 2011, AJ, 142, 184

Chen, B.-Q., Liu, X.-W., Yuan, H.-B. et al. 2014, MNRAS, 443, 1192

Cheng, J. Y., Rockosi, C. M., Morrison, H. L. et al. 2012a, ApJ, 752, 51

Cheng, J. Y., Rockosi, C. M., Morrison, H. L. et al. 2012b, ApJ, 746, 149

Costa, R. D. D., Uchida, M. M. M., Maciel, W. J., 2004, A\&A, 423, 199

Cui, X.-Q., Zhao, Y.-H., Chu, Y.-Q., et al. 2012, Research in Astronomy and Astrophysics, 12, 1197

Curir, A., Serra, A. L., Spagna, A. et al. 2014, ApJ, 784, 24

Daflon, S. \& Cunha, K., 2004, ApJ, 617, 1115

Dalcanton, J. J. \& Bernstein, R. A., 2002, AJ, 124, 1328

De Cat P., Fu J.-N., Yang, X.-H. et al. 2015, in preparation, preprint, arXiv:1411.0913

Demarque, P., Woo, J.-H.; Kim, Y-C. \& Yi, S. K., 2004, ApJS, 155, 667

Deng, L.-C., Newberg, H. J., Liu, C., et al. 2012, Research in Astronomy and Astrophysics, 12, 735

Dotter, A., Chaboyer, B., Jevremović, D., et al. 2008, ApJS, 178, 89

Elmegreen, B. G. \& Elmegreen, D. M., 2006, ApJ, 650, 644

Friel, E. D. \& Janes, K. A., 1993, A\&A, 267, 75

Friel, E. D. 1995, ARA\&A, 33, 381

Friel, E. D., Janes, K. A., Tavarez, M. et al. 2002, AJ, 124, 2693

Fuhrmann, K. 1998, A\&A, 338, 161

Fuhrmann, K. 2008, MNRAS, 384, 173

Gilmore, G. \& Reid, N., 1983, MNRAS, 202, 1025

Gilmore, G., Wyse, R. F. G. \& Kuijken, K., 1989, ARAA, 27, 555

Hayden, M. R., Holtzman, J. A., Bovy, J. et al. 2014, AJ, 147, 116

Haywood, M., Di Matteo, P., Lehnert, M. et al. 2013, A\&A, 560, 109 
Henden A. \& Munari U., 2014, CosKa, 43, 518

Henry, R. B. C., Kwitter, K. B., Jaskot, A. E. et al. 2010, ApJ, 724, 748

Ivezić, Ž., Smith, J. A., Miknaitis, G. et al. 2007, AJ, 134, 973

Jester S. et al., 2005, AJ, 130, 873

Jurić, M., Ivezić, Z., Brooks, A. et al. 2008, ApJ, 673, 864

Katz, D., Soubiran, C., Cayrel, R. et al. 2011, A\&A, 525, 90

Kazantzidis, S., Bullock, J. S., Zentner, A. R. et al., 2008, ApJ, 688, 254

Kazantzidis, S., Zentner, A. R., Kravtsov, A. V. et al., 2009, ApJ, 700, 1896

Kordopatis, G., Recio-Blanco, A., de Laverny, P. et al. 2011, A\&A, 535, 105

Kroupa, P. 2001, MNRAS, 322, 231

Kubryk, M., Prantzos, N. \& Athanassoula, E. 2013, MNRAS, 176, 31

Lacey, C. G. \& Fall, S. M., 1985, ApJ, 290, 154

Larson, R. B. 1976, MNRAS, 176, 31

Lee, Y.-S., Beers, T. C., An, D. et al. 2011, ApJ, 738, 187

Lemasle, B., François, P., Genovali, K. et al. 2013, A\&A, 558, bi31

Lépine, J. R. D., Cruz, P., Scarano, S. Jr. et al. 2011, MNRAS, 417, 698

Liu, X.-W., Yuan, H.-B., Huo, Z.-Y., et al. 2014, in Feltzing, S., Zhao, G., Walton, N., Whitelock, P., eds, Proc. IAU Symp. 298, Setting the scene for Gaia and LAMOST, Cambridge University Press, pp. 310-321, preprint (arXiv: 1306.5376)

Loebman, S. R., Roškar, R., Debattista, V. P. et al. 2011, ApJ, 737, 8

Luck, R. E., Gieren, W. P. \& Andrievsky, S. M. et al. 2003, A\&A, 2003, 401, 939

Luck, R. E., Kovtyukh, V. V., Andrievsky, S. M. et al. 2006, AJ, 132, 902

Luck, R. E., Andrievsky, S. M., Kovtyukh, V. V. et al. 2011, AJ, 142, 51

Luo A.-L., Zhao, Y.-H., Zhao, G. et al. 2015, Research in Astronomy and Astrophysics, in press, preprint (arXiv:1505.01570)

Maciel, W. J. \& Quireza, C., 1999, A\&A, 345, 629

Maciel, W. J., Costa, R. D. D., Uchida, M. M. M., 2003, A\&A, 397, 667

Maciel, W. J. \& Costa, R. D. D., 2009, in Andersen, J., Bland-Hawthorn, J., Nordström B., eds, Proc. IAU Symp. 254, The Galaxy disk in cosmological context, Cambridge University Press, p.38

Magrini, L., Sestito, P., Randich, S. \& Galli, D., 2009, A\&A 494, 95

Minchev, I., Famaey, B., Quillen, A. C. et al. 2012, A\&A, 548, 127

Munari U. et al. 2014, AJ, 148, 81

Nordström, B., Mayor, M., Andersen, J. et al. 2004, A\&A, 418, 989

Perryman M. A. C. et al., 1997, A\&A, 323, 49

Purcell, C. W., Kazantzidis, S. \& Bullock, J. S., 2009, ApJ, 694, 98

Quinn, P. J., Hernquist, L., Fullagar, D. P., 1993, ApJ, 403, 74

Read, J. I., Lake, G., Agertz, O. \& Debattista, V. P., 2008, MNRAS, 389, 1041

Reddy, B. E., Tomkin, J., Lambert, D. L. \& Allende Prieto, C., 2003, MNRAS, 340, 304

Reddy, B. E., Lambert, D. L. \& Allende Prieto, C. 2006, MNRAS, 367, 1329

Reid, M. J. 1993, ARA\&A, 31, 345

Robin, A. C., Reylé, C., Derrière, S. \& Picaud, S. 2003, A\&A, 409, 523

Roškar, R., Debattista, V. P., Quinn, T. R. et al. 2008, ApJ, 684, 79

Sánchez-Blázquez, P., Peletier, R. F., Jiménez-Vicente, J., et al. 2006, MNRAS, 371, 703

Schlegel, D. J., Finkbeiner, D. P. \& Davis, M. 1998, ApJ, 500, 525

Schlesinger, K. J., Johnson, J. A., Rockosi, C. M. et al. 2012, ApJ, 761, 160

Schlesinger, K. J., Johnson, J. A., Rockosi, C. M. et al. 2014, ApJ, 791, 112

Schönrich, R. \& Binney, J. 2009a, MNRAS, 399, 1145

Schönrich, R. \& Binney, J. 2009b, MNRAS, 396, 203

Soubiran, C., Bienaymé, O. \& Siebert, A., 2003, A\&A, 398, 141

Steinmetz, M., Zwitter, T., Siebert, A., et al. 2006, AJj, 132, 1645

Venn, K. A., Irwin, M., Shetrone, M. D. et al. 2004, AJ, 128, 1177

Villalobos, Á. \& Helmi, A., 2008, MNRAS, 391, 1806 
Xiang, M.-S., Liu, X.-W., Yuan, H.-B. et al. 2015a, MNRAS, 448, 90

Xiang, M.-S., Liu, X.-W., Yuan, H.-B. et al. 2015b, MNRAS, 448, 822

Yanny, B., Rockosi, C., Newberg, H. J. et al. 2009, AJ, 137, 4377

Yoachim, P. \& Dalcanton, J. J., 2008a, ApJ, 683, 707

Yoachim, P. \& Dalcanton, J. J., 2008b, ApJ, 682, 1004

Yong, D., Carney, B. W., Teixera de Almeida, M. L. \& Pohl, B. L., 2006, AJ, 131, 2256

Yuan H.-B., Liu X.-W. \& Xiang M.-S., 2013, MNRAS, 430, 2188

Yuan, H.-B., Liu, X.-W., Huo, Z.-Y. et al. 2015, MNRAS, 448, 855

Zacharias, N., Finch, C. T., Girard, T. M., et al. 2013, AJ, 145, 44

Zhao, G., Zhao, Y.-H., Chu, Y.-Q., Jing, Y.-P., \& Deng, L.-C. 2012, Research in Astronomy and Astrophysics, 12, 723

\footnotetext{
This paper was prepared with the RAA LATEX macro v1.2.
} 